\title{
Recognition of Textured Objects using Optimal Inverse Resonant Filtration
}

\author{
Roman Kvyetnyy,Olga Sofina, Yuriy Bunyak
}

\begin{abstract}
Recognition of textured objects is a typical problem in computer vision and pattern recognition. Usually are meeting two variations of this problem. The first is the recognition of a signed texture. The second is objects of interest recognition in image with textured background. We offer an approach for solving of the both problems with using of the inverse resonant filtration. A textured object or background dynamic space can be approximated by a set of principal eigenvectors in the form of resonantharmonic functions called as eigen harmonics (EH). The Inverse ResonantFilter (IRF) which is resonant in respect to EH series eliminates textured background and forms indicator signal of certain species. The IRF is founded on the approximation and extrapolation of the texture template signal by series of EH. Two methods of 2-dimensional (2D) EH parameter estimation are considered. The methods give estimates robust tobreaks and noise peakspresentedin textured image signal. The first method is based on the linear symmetry model and can be presented as a double linear prediction model. Additionally the condition of the unitary symmetry is used to provide stationarity and periodicity of the model. The second method is based on 2D correlation matrix splitting with projection into the subspace of principal harmonic components. Implementation of the IRF is considered in spatial and spectral domains. Discrete Fourier transform with eigen kernel (DFTEK) was used for design and realization of the high order IRF. The DFTEK has fractionally fast transform algorithm. Aligning image fragment phases improved inverse resonant filtration in the spectral domain. It was shown that the optimal approach to image filtration consists of an initial fast filtration in the spectral domain, followed by postfiltration of the image zones containing anomalous background variations using IRF in the spatial domain. It was shown that the IRF is invariant to shift transform. It can be invariant to affine and scale transform if instead initial texture and image are using their invariant pattern in the form of image surface geometry characteristics.
\end{abstract}

Index Terms:eigen-harmonic decomposition, inverse resonant filtration, pattern recognition

\section{Introduction}

Recognition of textured objects is one of the main challenges for visual based measurements and control systems. A texture can be an eliminating background or a target object. The problem is to define features which distinguish an image objects from their background. These may be statistical, structural and dynamic properties. The latest methods of texture analysis are examined in [1-4].

We will consider the methods of texture analysis from the point of view of their potential real time implementation by using arithmetic unit arrays such as Graphic Processor Units (GPU) and FPGA (Field-Programmable Gate Array).

The choice of method depends on the texture type - regular, quasi-regular, stochastic or dynamic. Most methods are based on statistical analysis, spectral transformations, and dynamic models of texture image signals. Our main interest is related with model based method of image analysis.

The statistical based methods are based on parametric and nonparametric models of the image textures such as Bayes analysis, Hidden Markov Models, Regression, Linear Autoregression (AR), Nonlinear Autoregression (NAR), and Autoregression with Moving Average (ARMA) [1-8]. The statistical nonparametric methods primary are nonlinear and not always give the textured image pattern estimates that are invariant in respect to spatial transforms of the image. 
Correlation methods can be implemented by arithmetic structures. The most widely used correlation method is the Method of Co-occurrence Matrices [2, 3, 9]. Further development of the correlation method includes definition of a nonlinear metric that relates with geometry of textures $[10,11]$.

The newest methods of object recognition are based on image signal transforms in the spatialfrequency domain, where spectral parameters of the image have simple statistics in a narrow range $[1,12]$. These methods combine statistical models and the Fourier transform. They use a set of functions of a certain type. The most popular are wavelet functions and their generalizations for $2 \mathrm{D}$ transforms [13],classic Gabor functions [14, 15].

The wavelet and Gabor spectrums represent local geometric features of textures with a minimal number of significant coefficients, however, they do not represent the texture periodicity because wavelets and Gabor functions have shift-variant spectrum. This problem was partially overcome by the introduction of the Complex Wavelet Transform (CWT) [16]. CWT transfers the spatial domain to frequency-phase domain and suppresses the spectrum aliasing that appears as a consequence of texture variations. CWT was also used to obtain the rotate-invariant transform [17]. Shift and rotate invariance have been investigated in some articles on object recognition.

A desire to present textures with a minimal number of parameters has driven the development of new transforms, many of which are a combination of existing transforms.

For example, the Radon transform in collaboration with common and wavelet based Fourier transforms provides the possibility to present images in a frequency-angle domain that has a much lower dimension as it is affine-invariant $[18,19]$.

Exact texture shape description is not necessary in many applications. When texture is dynamic the spatial and temporal changes are more important than local surface pattern characteristics.

Combining the eigenvector decomposition (EVD) of texture kernel with filters that resonant to certain image eigen modes gives most informative texture description [20]. This approach was widely developed in many papers[21-28]. The EVD is computationally complicated; therefore Independent Component Analysis (ICA) [21, 22], Empirical Mode Decomposition (EMD) [23-25] and Singular Values Decomposition (SVD) [26-28] are substituted for it. These decompositions reduce the size and dimension of texture parameters according to Principal Component Analysis (PCA).

PCA makes a projection of a textured image into a subspace configured by the most significant parameters. Usually the decompositions are used for full image transformation that complicates their application in real time systems.

Another implementation of PCA and EMD is based on the use of filters bank. Filters bank is synthesizing with the help of Fourier transform, wavelets and Gabor functions. The problems with texture analysis using filters bank arise from the sequence of tasks: decomposition; classification of the spectrum components; recognition.

Filtering procedures can be implemented using matrix transforms or convolution. This is done via components pattern analysis with thresholding, clustering, segmenting and modeling techniques [1-4].

In the case of dynamic textures decompositions are used dynamic models based on discrete differential equations [27-29]. The model synthesis is computationally complicated. But the model implementation is not complicated and can be executed in real time using a common signal processor or arithmetic array [28].

The correlation matrix and other integral matrices such as Wigner integral are used as objects of the decomposition because they represent the dynamic properties of a large image in compact form. The asymptotic correlation matrix of a stationary process has Toeplitz structure and its eigen vectors are harmonic in nature [30]. A textured image can be considered as a 2D stationary process with the block-Toeplitz correlation matrix. So, the harmonic decomposition is a natural 
approximation of EVD or EMD. The parameters of harmonics should agree with periods of a texture pixels and gray scale spatial variations. Such harmonics are considered as eigen or resonant with respect to texture structure.

The eigen-harmonic decomposition (EHD) is based on planar and spherical functions that have been used for compact presentation of textured images as well as human facial images in some articles [31, 32].

The demands of textured pattern recognition invariance are not restricted by shift and rotation invariance. These demands to textured object recognition are wider and can be formulated as follows.

The method should be invariant to scaling perturbations of target object in order to identify different resolutions of the same image objects.

The isometry invariance. This includes changes of brightness, rotations, mirror operations, color rotations and inversions.

Similarity. The recognition filters (methods) of similar images should be similar. To develop a notion of similarity one needs. Similarity of images may be measured by simple Euclidean distances.

The recognition filter should has a shorter representation than the associated object image.

These are the natural requirements if we are dealing with recognition filters (methods) of objects that are presented in metric spaces. The newest approach to invariant object recognition is the interpretation images as Riemannian manifolds [33]. In the context of image classification this is motivated by the fact that most content preserving operations are isometrics, i.e. this map and its inverse preserve the arclength of all curves [34]. This includes changes of brightness, rotations, mirror operations, color rotations and inversions.

The brief survey of textured image analysis methods allows the following conclusions:

- Dynamic model based methods of texture analysis and synthesis are the most effective. These methods can be used for analysis of static, dynamic, and quasi-regular textures. Models may be created with using a fragment of the image. The remainder of the image of an arbitrary size can be restored by the model operator using a generation process.

- Object recognition on a textured background may be accomplished by comparative analysis of the original and generated by the model images.

- An implementation of the structured object recognition using inverse in respect to object model schema yields to make recognition of the simple process that generate the texture by its model. Usually the generating process is white noise [27], so the recognition method may operate as a whitening filter similar to AR, NAR, and ARMA models. Variations of the retrieval generating signal can be described by the simple statistical parameters of mean value and dispersion. These parameters can serve as indicators of the target object.

- Autoregressive type models are not suitable for object recognition solutions because they reflect the frequency spectrum of the signal, but only indirectly reflect the amplitude spectrum by dumping factors of sinusoids. It is difficult to find a single valued solution for the estimation of whitening filter parameters because they relate to second order moments of texture signals. We will consider this problem solution based on linear inverse filtration which suppresses textured object structure and transforms it into simple for recognizing signal. At the same time it leaves foreign objects signal fluctuations without change of their energy. This type of inverse problem can be solved with using of the first order moments of textured image signals.

- The dynamic models are usually synthesized using the Fourier transform or decompositions mentioned above. The survey of previous research shows that the use of transforms and decompositions depends on the image type: full format, or only a typical fragment (known as texture kernel or as texton) [35]. When a full format image is using the wavelet and Radon transforms combined with Fourier transform give appropriate results. When a textonis using the 
wavelet transforms and SVD ignore the structural information because they are shift-variant. It has been shown that SVD and its multidimensional generalizationare effective for dynamic model synthesis $[27,28]$.

- The wavelet transforms have approximate and restricted shift invariance and cannot represent periodicity because they are intended for analysis of transient signals. EVD and EMD can represent texture periodicity as well as local kernel structure, however, the functional basis for these decompositions does not have analytical definition and is variant in respect to image shift. This fact complicates their use for interpolation of the image fragment when the exact texture period is unknown or periodically pulsating (such as a marine surface).

- In contrast to the mentioned decompositions, the EHD has simple analytical definition and join capabilities to approximation and interpolation of 2D fields [36]. Therefore the EHD is an appropriate function basis for the design of a linear inverse filter. The linear inverse filter suppress the approximation of texture structure in the form of eigen harmonic series. The harmonics in this series have resonant frequencies in respect to the image signal. An elimination of these harmonics makes the texture signal simpler and easier forrecognizing. This filter can be defined as the Inverse Resonant Filter (IRF).

Texture modeling and recognition with properties of invariance to space and scale perturbation can be made by including transforms of initial image into its invariant projections as surface curvature and Beltrami flow [33,34]. From the view of the EHD this approach is useful because the projections can transform mismatch for harmonic approximation step like image signal into sequence of fluctuations which associate with feature of step level change.

\section{Object recognition problem statement as the problem of optimal filtration}

We formulate theproblem of objects filteringas follows.

Let in the space $\Omega$ is given an image $X$ which includes an object $\mathrm{O}: \mathrm{O} \in \Omega_{\mathrm{O}} \subset \Omega$ as subset $\Omega_{\mathrm{O}}$ of $\Omega$. The aim isto define a linearornon-lineartransform

$$
F\left(\mathrm{O} ; x \in X: x \in \Omega_{\mathrm{O}}\right)=E
$$

where $E$ is a signal of object presence indicator. At the same time, the next condition should be satisfied together with (1.1):

$$
F\left(\mathrm{O} ; x \in X: x \notin \Omega_{\mathrm{O}}\right) \notin E .
$$

Recognition of some objects can be made using two conditions like (1.1) and (1.2):

$$
\begin{array}{ll}
F\left(\mathrm{O}_{i} ; x \in X: x \in \Omega_{\mathrm{O}_{i}}\right)=E ; & F\left(\mathrm{O}_{i} ; x \in X: x \notin \Omega_{\mathrm{O}_{i}}\right) \neq E \\
F\left(\mathrm{O}_{i} ; x \in X: x \in \Omega_{\mathrm{O}_{i}}\right)=E_{i} ; & F\left(\mathrm{O}_{i} ; x \in X: x \in \Omega_{\mathrm{O} k}\right) \neq E_{\forall k \neq i} .
\end{array}
$$

The condition (1.3) creates the filters of some different objects $\mathrm{O}_{i}$ without their classification. This condition is useful when the objects compile textured background and should be eliminated by filter. The condition (1.4) creates the filter for retrieval of the textured objects $\mathrm{O}_{i}$ with their classification. 
There are some approaches to problem (1.1) - (1.4) solution. They can be separated on two classes of methods - reversible and not reversible. Statistical and some spectral methods give the indicator Eparameters set not appropriate for initial object reconstruction compiles the second class. The deficiency of this methods class is absence of the feedback for control object changes by compare target object with its model. The feedback is inherent to reversible methods class. In this case it is meaning that there is inverse to filter $F(\cdot)$ operator $\Psi(\cdot)$ such that

$$
\Psi\left(\mathrm{O} ; E: E \in \Omega_{\mathrm{O}}\right) \cong X: X \in \Omega_{\mathrm{O}} .
$$

The expression (1.5) is the problem of the object modeling by using its characteristic $\mathrm{O}$ and indicator signal $E$. Evidently, this problem is inverse in respect to (1.1).

Object recognition characteristic relates with errors of the object model (1.5)

$$
X-\Psi(O ; E)=N(X)
$$

and filter (1.1)

$$
E-F(O ; X))=N(E)
$$

We can define the functional

$$
I=\underset{\Psi, F}{\arg \min }\left\{\int_{O}(X-\Psi(O ; E))^{2} d \Omega+\int_{O}(E-F(O ; X))^{2} d \Omega+\int_{O} \operatorname{Reg}(N ; F ; \Psi) d \Omega\right\},
$$

where $N(\cdot)$ is error noise, $\operatorname{Reg}(\cdot)$ is a regularization functional of additional restrictions on error shape (minimal variation or minimal surface). Euler-Lagrange variation of the functional (1.8) relatively parameters of the object filter and object model gives an optimal schema of objects recognition.

Let consider the schema $(1.1)-(1.8)$ when recognition filter and model filter are linear and are implemented as convolutions:

$$
\begin{aligned}
& \left.\widetilde{H}^{*} X=E: X \subset \Omega_{\mathrm{O}}\right) \\
& \left.H^{*} E=X: X \subset \Omega_{\mathrm{O}}\right)
\end{aligned}
$$

Variation of two first components in (1.8) with account of (1.9) and (1.10) yields the following expression.

$$
\left(X-H^{*} E\right) * E+(E-G * X) * X \approx 0
$$

where variation of the regularization functional is omitted. The $\delta$-function or Heaviside step function, uncorrelated white noise can be used as the object indicator. Then up to a constantfactor $H^{*} E(x, y) * E(x, y) \sim H$. The convolutions $E(x, y)^{*} X(x, y)$ are presented with different signs and therefore they can be eliminated. As a resultwe obtain the followingequation for optimal object filter and modeloperators characteristics.

$$
H-\widetilde{H}^{*} R_{X X} \cong 0
$$


where $R_{X X}$ - correlation matrix of the object signal. If it is satisfied the equation $\widetilde{H} * H \cong \mathrm{I}$, where I is the trivial operator, then convolution (1.12) with $H$ and $\widetilde{H}$ show that

and

$$
H * H \cong R_{X X}
$$

$$
I-\widetilde{H} * \widetilde{H} * R_{X X} \cong 0
$$

The expression (1.13) means that $H$ is object forming filter surge characteristic. It is well known as Wiener- Khintchine filter [37, 38]. This filter creates correlative relations of the object model by convolution with generating process in a manner of white noise or Heaviside step function. The expression (1.14) means that optimal recognition filter eliminates signal components which form correlative relations. By other words, filter is resonant with respect to object texture shape. Since it eliminates components we define it as inverse resonant filter. The expression (1.14) also show evident fact that the white noise which correlation has the form of I does not contain any object. The problem is to define principal components of an object texture which can be eliminated by simple linear convolution operation. The main demand to IRF properties is invariance to object space location and initial phase of its texture shape. This demand shortly can be formulated as shift invariance. It points on choice of a method for retrieval of principal components of the object model. The shift invariance is inherent to harmonical functions on condition that they are resonant with respect to object shape.

\section{Harmonic Model of Textured Image}

An ideal textured image of size $N \cdot P \times M \cdot Q$ can be represented as a tensor product of two matrices,

$$
D=T \otimes B
$$

where matrix $T$ of size $N \times M$ includes unit elements with insignificant fluctuations and matrix $B$ of size $P \times Q$ is the texture kernel. Matrix (1) rank equal to rank of the kernel matrix. Therefore it can be represented by the EVD and the SVD using $P$ left vectors and $Q$ right vectors. The same representation is possible using the Discrete Fourier Transform (DFT) in the basis of exponential functions [39]. The matrix $T$ can be decomposed as:

$$
T=F_{N} \operatorname{diag}\left[\begin{array}{llll}
1 & 0 & \ldots & 0
\end{array}\right] F_{M}^{H}
$$

where $F_{N}=1 / N\left[z_{i}^{k}\right]_{i, k=0 \ldots N-1} ; z_{i}^{k}=\exp (-\imath 2 \pi i k / N)$ is the DFT operator whose size is pointed bylow indexes. The kernel matrix has the following spectral decomposition:

$$
B=F_{P} A_{F B} F_{Q}^{H}
$$

where $A_{F B}$ is the spectral matrixof the kernel $B$ in the basis $F$. The substitution of expression (2) and (3) into (1) yields the following decomposition of the textured matrix 


$$
\begin{aligned}
& D=\left(F_{N} \otimes F_{Q}\right)\left(\operatorname{diag}\left[\begin{array}{llll}
1 & 0 & \ldots & 0
\end{array}\right] \otimes A_{F B}\right)\left(F_{Q}^{H} \otimes F_{M}^{H}\right)= \\
& F_{N P} \operatorname{diag}\left[\begin{array}{llll}
A_{F B} & 0 & \ldots & 0
\end{array}\right] F_{M Q}^{H} .
\end{aligned}
$$

In the expression (4) was used associativity of the tensor product and that $F_{N P}=F_{N} \otimes F_{P}$, $F_{M Q}=F_{M} \otimes F_{Q}$. It shows that the DFT is informative as EVD and SVD on condition the basis is exactly aligned with texture structure. Additionally, the DFT representation (4) is invariant with respect to image initial point shift. The textured matrix (1) initial point causes the cyclical shift of the kernel matrices. The shifted matrix relates with the original matrix $B$ by simple expression

$$
B^{t, \tau}=C_{P}^{t} B C_{Q}^{\tau H}
$$

where $B^{t, \tau}$ - shifted matrix along $t$ rows and $\tau$ columns, $C_{P}$ - cyclical shift operator (CSO) of size $P \times P$ [39]. The following expression is the well-known CSO spectral decomposition:

$$
\begin{aligned}
& C_{P}^{t}=F_{P} \operatorname{diag}\left[z_{1}^{t} \ldots z_{P}^{t}\right] F_{P}^{H} \\
& C_{Q}^{\tau}=F_{Q} \operatorname{diag}\left[z_{1}^{\tau} \ldots z_{Q}^{\tau}\right] F_{Q}^{H} .
\end{aligned}
$$

The texture matrix (1) with shifted kernel matrices (5) can be represented with account of expressions (3) - (6) as

$$
\begin{aligned}
& D^{t, \tau}=T \otimes B^{t, \tau}= \\
& F_{N P} \operatorname{diag}\left[\operatorname{diag}\left[z_{1}^{t} \ldots z_{P}^{t}\right] A_{F B} \operatorname{diag}\left[z_{1}^{\tau^{*}} \ldots z_{Q}^{\tau^{*}}\right] \quad 0 \quad \ldots 0\right] F_{M Q}^{H}
\end{aligned}
$$

where * is a complex conjugate. The spectral factorization of the textured image matrix (4) and (7), shows that the power spectrum of the kernel matrix is invariant to transforms by the CSO (6) because the spectrum matrix of the shifted kernel (5) differs from the original by phase multiplies.

The main problemof spectral factorization in canonical manner, as shown in (4) and (7), is the alignment of the DFT with texture structure. There are known methods of texture period estimation based on algebraic geometry and complex EMD [40,41]. Another method is based on adaptation of the EHD to texture structure, idealized or real. This adaptation can be made by determining the shift transform that is natural for a given texture [27]. The eigen vectors system of the shift transform can then serve as a generalized harmonical basis for texture presentation. Following this approach we substitute linear shift instead of cyclic shift (5). This operation transfers rows (or columns) of the image matrix linearly on one step. Each new row or column is a linear combination of adjacent rows(columns). The new rows and columns are the approximate model of real image rows and columns that appear as a result of the image shift. The Linear Shift Operator (LSO) has the form of a Frobenius matrix 


$$
K_{P}=\left[\begin{array}{cccc}
0 & 1 & \cdots & 0 \\
\vdots & \vdots & \ddots & \vdots \\
0 & 0 & \cdots & 1 \\
-a_{P} & -a_{P-1} & \cdots & -a_{1}
\end{array}\right]
$$

and its characteristic polynomial

$$
1+\sum_{i=1}^{P} a_{i} z^{i}=0
$$

has the roots $z_{i=1 \ldots P}$ that have exponential form in analytical presentation: $z_{i}=\exp \left(\imath 2 \pi f_{i}\right)$, where $f_{i}$ - eigen or resonant frequencies. The LSO (7) can be defined for two image coordinates. The spectral factorization of the LSO (8) is similar to (6) and can be written for both coordinates as

$$
\begin{aligned}
& K_{x P}^{t}=Z_{x P} \operatorname{diag}\left[z_{x 1 \ldots}^{t} \ldots z_{x P}^{t}\right] Z_{x P}^{\#} ; \\
& K_{y Q}^{\tau}=Z_{y Q} \operatorname{diag}\left[z_{y 1 \ldots}^{\tau} \ldots z_{y Q}^{\tau}\right] Z_{y Q}^{\#},
\end{aligned}
$$

where $Z_{x P}=\left[z_{x i}^{t}\right]_{i=1 \ldots P}^{t=0 . . P-1}, Z_{y Q}=\left[z_{y i}^{t}\right]_{i=1 \ldots Q}^{t=0 . . Q-1}$, \# is the pseudoinverse. The shifted linear kernel matrix can be presented by the EHD in the following manner,

$$
\begin{aligned}
& B^{t, \tau}=K_{P}^{t} B K_{Q}^{\tau T}= \\
& Z_{x P} \operatorname{diag}\left[z_{x 1 \ldots}^{t} \ldots z_{x P}^{t}\right] Z_{x P}^{\#} Z_{x P} A_{Z B} Z_{y Q}^{T} Z_{y Q}^{\# T} \operatorname{diag}\left[z_{y 1 \ldots}^{\tau} \ldots z_{y Q}^{\tau}\right] Z_{y Q}^{T}= \\
& Z_{x P} \operatorname{diag}\left[z_{x 1 \ldots}^{t} \ldots z_{x P}^{t}\right] A_{Z B} \operatorname{diag}\left[z_{y 1 \ldots}^{\tau} \ldots z_{y Q}^{\tau}\right] Z_{y Q}^{T},
\end{aligned}
$$

where $T$ is the transposition, and $A_{Z B}$ is the spectral matrix of the matrix $B$ in the basis $Z$.

$$
A_{Z B}=\widetilde{Z}_{x P}^{\# B} \widetilde{Z}_{y Q}^{\# T}
$$

Expression (11) shows the spectrum invariance to linear shift operations that is equivalent to expression (7). The kernel periodicity in a textured image is defined by the condition

$$
B^{m P, n Q}=B
$$

where $m$ and $n$ are arbitrary integer values. This condition allows presentation of the full image $D$ by overdetermined matrices $\widetilde{Z}_{x P}=\left[z_{x i}^{t}\right]_{i=1 \ldots P}^{t=0 \ldots N P-1}, \widetilde{Z}_{y Q}=\left[z_{y i}^{t}\right]_{i=1 \ldots Q}^{t=0 \ldots M Q-1}$ using the spectral matrix $(2.12)$, 


$$
D \approx \widetilde{Z}_{x P} A_{Z B} \widetilde{Z}_{y Q}^{T} .
$$

Expression (14) is analogous to (4) but it is approximate because the pseudoinverse of the overdetermined matrices yields approximate results.

Linear shift is natural for many models of textured images, both static and dynamic. Therefore the EHD provides appropriate representation of the image model. The parameters of the OLS (8) should be estimated in accordance with the dynamic properties of texture. As it follows from condition (13), the shift operations do not change the texture signal energy. Therefore, the shift operator is unitary. The operator (8) is unitary if its characteristic polynomial (9) roots lie on the unit circle in the complex domain. It is important the problem to define the LSO order $P$ and $Q$ for both coordinates.

The spectral presentation (14) is basing on the linear shift model of the data matrix in the form of two LSO (8) associated with the coordinates. The roots of the characteristic polynomial (9) are using for generation of the functions basis of the discrete Fourier transform. Such spectral presentation may be used if the texture is homogeneous. In other case estimation of the EHD component should accounts full texture image matrix. For this aim may be used 2D generalization of the LSO

$$
K_{P \times Q}^{(2)}=K_{x P} \otimes K_{y Q} \quad .
$$

The evaluation of the 2D LSO parameters in the manner of product of two polynomials associated with coordinates has nonlinear feature [42]. As it was shown in [43], the parameters of the characteristic polynomials (9) associated with the coordinates and approximation of the polynomials product can be estimated by using linear extended equations based on the LSO (15). The roots of the polynomials products are useful for definition of the 2D harmonics parameters pares.

\section{Optimal Inverse Resonant Filter Design}

Linear version (1.9) of the object recognition schema (1.1) is the inverse problem with respect to object signal model in the manner of forming filter (1.10). It means restoration of the originating signal matrix $E=\left[e_{i, k}\right]_{i=0 . \ldots M-1}^{k=0 . N-1}$ using measurement data $D=\left[d_{i, k}\right]_{i=0 . \ldots M-1}^{k=0 . N-1}$ of size $M \times N$ in the object region of the image under the assumption that mentioned matrices are connected by some linear operator with unknown transient characteristic $H=\left[h_{m, n}\right]_{m=0 . . P-1}^{n=0 . Q-1}$. The measured data matrix includes samples of additive noise $\xi_{i, k}$ associated with texture model error. Formally the model can be written in the discrete manner as the convolution equation

$$
d_{i, k}=\sum_{m=0}^{P-1} \sum_{n=0}^{Q-1} h_{m, n} e_{i-m, k-n}+\xi_{i, k} .
$$

There is classical Wiener solution of the inverse problem for the model (1) using the Fourier transform [30,37]. However, this solution assumes that noise is observed separately and the statistical parameters of the image are known. If noise is not significant it is usually ignored. When noise is not used the Wiener approach may give unstable or singular solutions. Methods of regularization are used to overcome this problem. The modern methods of regularization are based on the PCA with SVD or EMD of the measured data. 
The equation (1) can be considered as a linear dynamic model of the textured object image $D$ which is initiated by the generating process $E$ of known form. This model is similar to those ones that were considered in [27-29]. The generating process is the object presence indicator. It should have simple form which is easy for recognizing.

The filter has a much smaller size than the object image size. Therefore the problems of the direct and inverse transient response evaluation are ill posed and need the regularization. We offer to consider the EHD as the PCA and as the basis of the Fourier transform in (2.13) for design the inverse filter and its regularization simultaneously.

Let the 2D Heaviside step function

$$
e_{i k}=\left\{\begin{array}{l}
1, \quad\left(i \geq 0^{+}\right) \wedge\left(k \geq 0^{+}\right) \\
0.5, \quad i=0, k=0 \\
0, \quad\left(i \leq 0^{-}\right) \vee\left(k \leq 0^{-}\right),
\end{array}\right.
$$

is the generating process in (1). The textured object image is specified in range $\left(i \geq 0^{+}\right) \wedge\left(k \geq 0^{+}\right)$ and is a response of linear dynamic system (1) to the excitation (2). Let us consider the problem of generating signal reconstruction using an inverse filter with transient characteristic $\widetilde{h}_{m, n}$. The generating signal has constant value $E$ in the range of image, so the problem appears in the following way:

$$
\sum_{m=0}^{P-1} \sum_{n=0}^{Q-1} \widetilde{h}_{i+m, k+n} d_{m, n}=E+\varsigma_{i, k},
$$

where $\varsigma_{i, j}$ represents error noise. The dispersion value of the noise in (3) serves as a criterion for filter matching with object texture.

There are some approaches to solving the problem (3). We can compile the system of equations (3) for shifted data matrices

$$
\sum_{m=0}^{P-1} \sum_{n=0}^{Q-1} \widetilde{h}_{i+m, k+n} d_{m+t, n+\tau} \cong E: \forall d_{m+t, n+\tau} \in O \subset \Omega
$$

and find minimum square solution of the system (4) with respect to transient characteristic $\widetilde{h}_{m, n}$ elements by pseudoinverse of the extended data matrix. But such solution will be not shift invariant. It obtains the shift invariance on condition that it is presented in shift invariant functions basis.

Let the elements of the object image matrix are presented by EHD (2.13) as the following series:

$$
d_{i, k}=\sum_{m=1}^{P} \sum_{n=1}^{Q} A_{m, n} z_{x m}^{i} z_{y n}^{k}+\varepsilon_{i, k}
$$

where $A_{m, n}$ represents the amplitudes of the two-dimensional harmonic functions $z_{x m}^{i} z_{y n}^{k}, \varepsilon_{i, k}$ is the approximation error which has muchless dispersion than signal power. The resonant frequencies $f_{x m}, f_{y n}$ are the main parameters of the harmonic functions $z_{x m}^{i}=\exp \left(\imath 2 \pi f_{x m} i\right)$ and $z_{y n}^{k}=\exp \left(22 \pi f_{y n} k\right)$ in (5). Their values characterize eigen fluctuations of the target object surface 
associated with the coordinate axes $O X$ and $O Y$. This frequencies set relates with roots of characteristic polynomial (2.9) of the texture dynamic model (1).

It is not necessary to use a full textured object image for series (5) parameters determination. Some base region $D: B \subset D$ of the size $n_{x} \times n_{y}: n_{x}>2 P ; n_{y}>2 Q$ is sufficient. This region should covers the texture kernel $B$. The EHD of the base region is equivalent to (2.13) and it can be represented in the matrix form as

$$
\begin{aligned}
& D \approx Z_{x} A Z_{y}^{T} \\
& A=Z_{x}^{\#} D Z_{y}^{T \#},
\end{aligned}
$$

where $A=\left[A_{i k}\right]_{i=1 \ldots P}^{k=1 . . . Q}, Z_{x(y)}=\left[\begin{array}{c}z^{k} \\ x(y) i\end{array}\right]_{i=1 \ldots P(Q)}^{k=0 \ldots n_{x(y)}-1}$.

Let point attention to shift invariance of the direct and pseudo-inverted EHD bases because it is important for the spectral presentation (6) - (7). The $t$-shifted along space coordinate matrix $Z_{x}^{t}=Z_{x} \operatorname{diag}\left[z_{x 1}^{t} \ldots z_{x P}^{t}\right]$ in pseudo-inverted manner has the form of $t$-shifted matrix $Z_{x}^{\# t}=\operatorname{diag}\left[z_{x 1}^{-t} \ldots z_{x P}^{-t}\right] Z_{x}^{\#}$ too. So, the pseudo-inverted matrix $Z_{x}^{\# t}=\operatorname{diag}\left[z_{x 1}^{-t} \ldots z_{x P}^{-t}\right] Z_{x}^{\#}$ is shift invariant too.

By analogy to (6) - (7), we find the EHD of transient characteristic,

$$
h_{i, k}=\sum_{m=0}^{P-1} \sum_{m=0}^{Q-1} H_{m, n} z_{x m}^{i} z_{y n}^{k}
$$

The substitution equations (5) - (8) into (1) with neglecting the error yields the following spectral decomposition.

$$
\begin{aligned}
d_{i, k} \cong & \sum_{m=1}^{P} \sum_{n=1}^{Q} \sum_{t=0}^{P-1} \sum_{\tau=0}^{Q-1} H_{m, n} z_{x m}^{i+t} z_{y n}^{k+\tau} e_{t, \tau}= \\
& \sum_{m=1}^{P} \sum_{n=1}^{Q} H_{m, n} z_{x m}^{i} z_{y n}^{k} \sum_{t=0}^{P-1} \sum_{\tau=0}^{Q-1} e_{t, \tau} z_{x m}^{t} z_{y n}^{\tau}= \\
& \sum_{m=1}^{P} \sum_{n=1}^{Q} H_{m, n} E_{m . n} z_{x m}^{i} z_{y n}^{k},
\end{aligned}
$$

where

$$
E_{m, n}=\sum_{i=0}^{P-1} \sum_{k=0}^{Q-1} e_{i, k} z_{x m}^{i} z_{y n}^{k}=\sum_{i=0}^{P-1} \sum_{k=0}^{Q-1} z_{x m}^{i} z_{y n}^{k}
$$

in accordance with (2). As it follows from expressions (1), (9), $A_{m, n} \approx H_{m, n} E_{m, n}$ and the IRF spectrum

$$
\widetilde{H}_{m, n} \cong H_{m, n}^{-1}=E_{m, n} A_{m, n}^{-1}
$$


The transient characteristic of the IRF (3) for object texture recognition can be defined using equation

$$
\left.\widetilde{H}=\mid \widetilde{h}_{i, k}=\sum_{m=1}^{P} \sum_{n=1}^{Q} E_{m, n} A_{m, n}^{-1} z_{x m}^{i} z_{y n}^{k}\right\rfloor_{i=0 \ldots P-1 ; k=0 \ldots Q-1} .
$$

The schema (6) - (12) implements the approach to filter design basing on Fourier transform. It differs from the well-known classic schema by using EHD instead usual discrete Fourier transform. The EHD allows avoid of small amplitudes $A_{m, n}$ influence because all eigen fluctuations have comparative value levels. This creates the effect of the regularization of the solution (12).

In the given above schema the base equations (5), (8) and (9) are approximate. So, this approach gives an estimate of the aim transient characteristic. It needs optimization in accordance with a criterion of quality. The main factor of an influence is variation of data spectrum because the recognizing objects are only similar to template object which was used for filter design. As the elements of $E_{m, n}$ in (10) are not varied by data fluctuations directly the better way is to define the transient characteristic of the forming filter as $H_{m, n} \cong E_{m, n}^{-1} A_{m, n}$ and then evaluate the inverse filter using the condition

$$
\widetilde{\mathbf{h}}^{T} \cdot \mathbf{H} \cong[0 \ldots 010 \ldots 0],
$$

where $\mathbf{H}$ is the matrix which is equivalent to convolution operation with vector $\widetilde{\mathbf{h}}$.

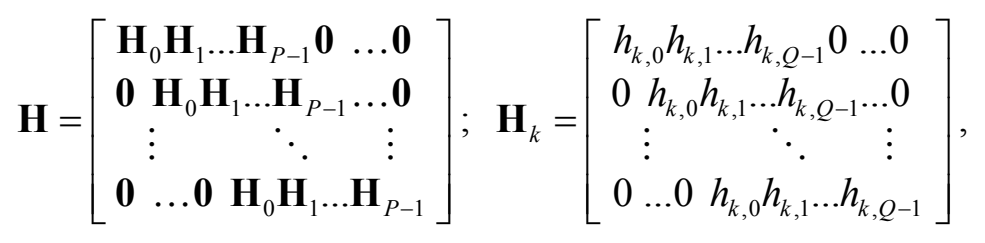

where $\mathbf{0}$ - the null matrix. The vector $\widetilde{\mathbf{h}}$ is the lexicographical presentation of the matrix $\widetilde{H}$ (12). The solution of the (13) we can find by the method of minimum squares [30].

$$
\widetilde{\mathbf{h}}^{T} \cong\left(\mathbf{H} \cdot \mathbf{H}^{T}\right)^{-1}(\mathbf{H})_{M},
$$

where $M=(2 P Q-1) / 2$.

The approximate solution (15) is not optimal because it is not shit invariant and includes fluctuations which are enforced by operation of inversion.

Using (13) and (14) we can define the functional for the IRF spectrum optimization.

$$
I_{\widetilde{h}}=\underset{\widetilde{H}}{\arg \min }\left\{\begin{array}{l}
\sum_{i, k \in O}\|\| \sum_{m=1}^{P} \sum_{n=1}^{Q} \sum_{t=0}^{n_{x}-P} \sum_{\tau=0}^{n_{y}-Q} \widetilde{H}_{m, n} z_{x m}^{i+t} z_{y n}^{k+\tau} h_{t, \tau}-\delta_{i, i_{0}} \delta_{k, k_{0}} \|^{2}+ \\
\lambda \cdot \operatorname{Reg}(\widetilde{H})
\end{array}\right\},
$$


where $\left(i_{0}, k_{0}\right)$ is a central point in object region, $\operatorname{Reg}$ and $\lambda$ are regularization functional and parameter, which set conditions for additional restrictions on the function $\widetilde{H}$ surface. As the regularization functional can be used the area of the surface $\widetilde{H}$ with the aim to minimize it. This condition provides minimization of fluctuations and serves main part of the characteristic feature. The surface area of the function $H(x, y)$ is defined as [33]

$$
\Sigma_{H}=\int_{O} \sqrt{1+H_{x}^{2}+H_{y}^{2}}(x, y) d x d y
$$

where $H_{x}=\partial H(x, y) / \partial x$ and $H_{y}=\partial H(x, y) / \partial y$. In the spectral domain the surface area (17) as the regularization functional will be of the following form:

$$
\operatorname{Reg}(\widetilde{H})=\sum_{i, k \in O} \sqrt{1+\left(\sum_{m, n} \widetilde{H}_{m, n} d z_{x m} z_{x m}^{i} z_{y n}^{k}\right)^{2}+\left(\sum_{m, n} \widetilde{H}_{m, n} d z_{y m} z_{x m}^{i} z_{y n}^{k}\right)^{2}}
$$

where $d z_{x(y) m}=0.5\left(z_{x(y) m}-z_{x(y) m}^{-1}\right)$ are spectrum coefficients introduced by discrete derivatives of the harmonic functions. Euler-Lagrangevariational derivativeof the functional (16) and surface area (18) looks as the following equation (all indexes serve numbering like in (16)).

$$
\begin{aligned}
\frac{\partial I_{\widetilde{h}}}{\partial \widetilde{H}_{u, v}}= & \sum_{m, n} \widetilde{H}_{m, n}\left(2 \sum_{i, k \in O} \sum_{t, \tau} h_{t, \tau} \sum_{i, k \in O} z_{x m}^{i+t} z_{y n}^{k+\tau} \sum_{t^{\prime}, \tau^{\prime}} h_{t^{\prime}, \tau^{\prime}} z_{x u}^{i+t^{\prime}} z_{y u}^{k+\tau^{\prime}}-\right. \\
& \left.\lambda\left(d z_{x m} d z_{x u}+d z_{y n} d z_{y v}\right) \sum_{i, k \in O} z_{x m}^{i} z_{y n}^{k} z_{x u}^{i} z_{y v}^{k} \operatorname{Reg}(\widetilde{H})^{-1}\right)_{-} \\
& \sum_{i, k \in O} \sum_{t^{\prime}, \tau^{\prime}} h_{t^{\prime}, \tau^{\prime}} z_{x u}^{i+t^{\prime}} z_{y u}^{k+\tau^{\prime}}=0 .
\end{aligned}
$$

The $P Q$ expressions (19) create the system of equations for iterative evaluation of the IRF transient characteristic optimal spectrum $\widetilde{H}$ elements,

$$
\sum_{m, n} \widetilde{H}_{m, n}^{(p)} \Psi_{u, v ; m, n}\left(\widetilde{H}^{(p-1)}\right)=\psi_{u, v} ; u=0, \ldots, P-1 ; v=1, \ldots, Q-1,
$$

where

$$
\begin{gathered}
\Psi_{u, v ; m, n}=\left(2 \sum_{i, k \in O} \quad \sum_{t, \tau} h_{t, \tau} \sum_{i, k \in O} z_{x m}^{i+t} z_{y n}^{k+\tau} \sum_{t^{\prime}, \tau^{\prime}} h_{t^{\prime}, \tau^{\prime}} z_{x u}^{i+t^{\prime}} z_{y u}^{k+\tau^{\prime}}-\right. \\
\left.\lambda\left(d z_{x m} d z_{x u}+d z_{y n} d z_{y v}\right) \sum_{i, k \in O} z_{x m}^{i} z_{y n}^{k} z_{x u}^{i} z_{y v}^{k} \operatorname{Reg}\left(\tilde{H}^{(p-1)}\right)^{-1}\right), \\
\psi_{u, v}=\sum_{i, k \in O} \sum_{t, \tau} h_{t, \tau} z_{x u}^{i+t} z_{y u}^{k+\tau}
\end{gathered}
$$

$\widetilde{H}_{m, n}^{(p)}$ is the result of $p$-th iteration step, $\widetilde{H}_{m, n}^{(0)}=\widetilde{H}_{m, n}$ in (11) or $\widetilde{H}_{m, n}^{(0)}$ can be obtained by EHD of the (15). The solution of the (20) looks as 


$$
\widetilde{H}_{u, v}^{(p)}=\sum_{m, n} \Psi_{u, v ; m, n}^{-1}\left(\widetilde{H}^{(p-1)}\right) \psi_{m, n} ; u=0, \ldots, P-1 ; v=1, \ldots, Q-1
$$

The regularization parameter $\lambda$ can be chosen by the help of the condition of convergence of the $q$ first iterations:

$$
\sum_{u, v=0}\left\|\widetilde{H}_{u, v}^{(t+1)}-\widetilde{H}_{u, v}^{(t)}\right\|^{2} \theta \leq \sum_{u, v}\left\|\widetilde{H}_{u, v}^{(t)}-\widetilde{H}_{u, v}^{(t-1)}\right\|^{2}
$$

where $t=1,2, . ., q, \theta-$ a positive value The iteration process can be stopped if

$$
\sum_{u, v}\left\|\tilde{H}_{u, v}^{(t+1)}-\widetilde{H}_{u, v}^{(t)}\right\|^{2} \leq \varepsilon_{\widetilde{H}}
$$

where $\varepsilon_{\widetilde{H}}$ is a small value.

The optimization of the IRF transient characteristic may be made in space coordinates. But in this case on each step of iterations the operation of projection in to EHD spectrum space is necessary for serving of the IRF shift invariance. The projection consists in direct and inverse Fourier transforms (7) and (6). Because the basis of EHD is not complete this operation is not trivial.

The next step of the optimal IRF transient characteristic evaluation is its normalization in accordance with the condition:

$$
\widetilde{\mathbf{h}}^{T} \cdot \mathbf{H} \cong \mathbf{h}^{T} \cdot \widetilde{\mathbf{H}}
$$

Let multiply both parts of (24) by $\mathbf{H}^{T}$. Then as well as in (15) we can define the vector

$$
\widetilde{\mathbf{h}}^{(k+1) T}=\mathbf{h}^{(k) T}\left(\widetilde{\mathbf{H}} \cdot \mathbf{H}^{T}\right)\left(\mathbf{H} \cdot \mathbf{H}^{T}\right)^{-1} .
$$

The obtained vector in (25) can be considered as the next in the series of iterations. The right part of the expression (25) includes the matrix $\widetilde{\mathbf{H}}$ that is compiled with using of the previous solution vector. The iterative process is continuing up to the condition

$$
\sum_{i, k}\left\|\widetilde{h}_{i, k}^{(t+1)}-\widetilde{h}_{i, k}^{(t)}\right\|^{2} \leq \varepsilon_{\widetilde{h}}
$$

will be met $\left(\varepsilon_{\widetilde{h}} \sim 10^{-8} \ldots 10^{-10}\right)$.

The procedures of optimization in spectral domain and presented above procedure of the normalization can be rearranged in their sequence. The equations (16) and (25) show that in case of normalization the influence of the forming filter transient characteristic is more significant then in the case of the optimization in spectral domain. So, if the forming filter is given with high level of trust then the normalization should be the last operation. If not then it should be first one or omitted.

The alternative to the IRF is so called auto-regression with shifted mean (ARSM) [30] which can be written as 


$$
\sum_{m=1}^{P} \sum_{n=1}^{Q} a_{m, n} d_{i-m, k-n}=d_{i, k}+E+\varsigma_{i, k},
$$

where $a_{m, n}$ are the parameters of the model, $E$ is the shift of the mean of the error noise $\varsigma_{i, k}$. It is evident similarity of the models (3) and (27). The ARSM is shift invariant. This follows from its definition form. In general, it can not be presented as series of harmonics. This differs it from the IRF. We will study this difference with using examples of object recognition below.

\section{Creation of the Functions Basis for Eigen Harmonic Decomposition}

The first step of the IRF design is the EHD of the target textured object template. There are known some approaches to $2 \mathrm{D}$ nonlinear spectral analyses. The simplest way is the estimation of the LSO (2.8) parameters and its characteristic polynomial (2.9) roots for two space coordinates. This approach gives appropriate result if the 2D signal is uniform. If the signal is not uniform, the full matrix of 2D data should be used. The following approaches to 2D EHD are developed: 2DMUSIC (Multiple Signal Classification) [30], 2D-ESPRIT (Estimation of Signal Parameter via Rotational Invariance Techniques) [44], 2D-Matrices Pencil Method (MPM) [45], Total Least Squares (TLS) version of MPM [46], and 2D-Linear Prediction Method (LPM) using the 2D generalization of the LSO $(2.8)$ as $(2.14)[42,43]$. In the case of texture EHD, it is necessary to take into account important restrictions to the model parameters:

- The texture model is considering as a response on excitement (3.2) of the linear dynamic system (3.1). Texture stationarity implies the condition of periodicity which is defined by (2.12). Therefore, the eigen modes in (3.5) should have a trivial dumping factor. This imply that the characteristic polynomial (2.9) roots of the model must be placed on the unit circle. In other words, the dynamic model must have unitary symmetry in respect to the linear shift operations.

- Texture might not be a smooth function. Therefore the method of the EHD should not be sensitive to image signal breaks.

- The inverse problem solution in spectral domain of the EHD requires the determination of the principal eigen mode numbers to exclude unstable results.

- A high order filter is required when background texture is complicated, quasi-regular, or corrupted by noise spikes.

The last two requirements above are mutually conflicting when used to determine the IRF order. This conflict requires the design of a spectral basis adapted to the specific image signal properties if high order inverse filtration is to be performed.

\section{A. Estimation of Texture Resonant Spectrum}

The methods of spectral parameter estimation that we have examined are based on a correlative relation between two mutually shifted data sets. Linear parameterization of this relation yields the linear prediction (LP) model and its various implementations. LP based methods are sensitive to signal phase. Usually this sensitivity becomes apparent in splitting of spectral lines[30]. Methods that are not sensitive to image signal phase brake must be designed. We will consider an approach based on 1D data harmonic decomposition, and an approach based on 2D data harmonic decomposition.

The linear symmetry method 
The product of sample matrices $X_{0}=\left[x_{i+k}\right]_{i=0 \ldots P-1, k=0 \ldots . N-P-1}$ and $X_{0}^{T}$ forms the correlation matrix of size $P \times P$

$$
R_{P}=X_{0} X_{0}^{T}
$$

The same product of matrices $X_{1}=\left[x_{i+k+1}\right]_{i=0 \ldots P-1, k=0 \ldots . N-P-1}$ and $X_{1}^{T}$.gives the correlation matrix too. These matrices and matrices in (1) are related by OLS (2.8):

$$
X_{1} X_{1}^{T}=K_{P} X_{0} X_{0}^{T} K_{P}^{T}=K_{P} R_{P} K_{P}^{T} .
$$

If data set is infinitely long then the product (2) is equal to (1). So, we have the equation

$$
R_{P}=K_{P} R_{P} K_{P}^{T}
$$

which define the Linear Symmetry (LS) [47] of the correlation matrix. The usual LP model like (3.27) is shift invariant relatively data first moments in the manner of data samples. The LS supplement the LP model by the condition of invariance to shift operations of the second moments - correlation samples. This restriction on the data model is imposed with the aim to reduce sensitivity to data breaks. As it was shown in [47] this additional symmetry removes LP model dependence on signal initial phase. Equation (3) providesunitarityof the LSO because the similarity transformation (3) does not change energy of the correlation matrix. It can be easy proved that transformation (3) is valid on condition that correlation matrix has Toeplitz structure. This allows to use the correlation function for evaluation model LS parameters as well as in Levinson-Durbin algorithm [30]. Though, when data set is short the Toeplitz structure corrupts spectrum. Therefore we consider the solution of the LS equation which gives unitarity LSO and uses the correlation matrix (1).

Let write expression of the last row and column transforms in equation (3) on condition the correlation matrix is Toeplitz and is signed by correlation function vector $\left[r_{i}\right]_{i=0 . . . P-1}$. To simplify the expressions we will use the following designations: $\alpha_{i}=-a_{P-i}, i=0 \ldots P-1$. Then the equation for first $p-1$ elements has the next form.

$$
\sum_{i=0}^{k} r_{k-i+1} \alpha_{i}+\sum_{i=0}^{P-k-2} r_{i} \alpha_{i+k+1}=r_{P-1-k},
$$

where $k=0 \ldots p-2$. The equation for the last element is the following.

$$
\sum_{k=0}^{P-1} \alpha_{k}\left(\sum_{i=0}^{k-1} r_{k-i} \alpha_{i}+\sum_{i=0}^{P-k-1} r_{i} \alpha_{i+k}\right)=r_{0} .
$$

The system of equations (4) - (5) contains the nonlinear equation (5) relatively of $P^{2}$ squares of the desired values. Therefore it does not have unique solution. One of them can be found by the approach basing on the reference element method.

The reference element should be that which is most sensitive to influence of the polynomial (2.9) roots. It is known that 


$$
-a_{1}=\alpha_{P-1}=\sum_{i=1}^{P}\left(z_{i}+\delta z_{i}\right) ;-a_{P}=\alpha_{0}=\prod_{i=1}^{P}\left(z_{i}+\delta z_{i}\right)
$$

If $\left|z_{i}\right| \leq 1$ then it is evident from (6) that $-a_{1}=\alpha_{P-1}$ is mostly sensitive to roots variations $|\delta z|<<1$ because the rest coefficients are combinations of sums of multiplicative components. The multiplicative components include second other variations and weighted variations.

Let select in $P-1$ equations (4) the components related with $\alpha_{P-1}$ and carry them from the left part to the right part. The obtained equations system in the matrix form can be written as

$$
\left[\begin{array}{cccc}
r_{1} & r_{0} & \cdots & r_{P-3} \\
r_{0} & r_{1} & \cdots & r_{P-4} \\
\vdots & \vdots & \ddots & \vdots \\
r_{P-3} & r_{P-4} & \cdots & r_{1}
\end{array}\right] \cdot\left[\begin{array}{c}
\alpha_{0} \\
\alpha_{1} \\
\vdots \\
\alpha_{P-1}
\end{array}\right]=\left[\begin{array}{c}
r_{P-1}-\alpha_{P-1} r_{P-2} \\
r_{P-2}-\alpha_{P-1} r_{P-3} \\
\vdots \\
r_{1}-\alpha_{P-1} r_{0}
\end{array}\right]
$$

The Toeplitz matrix in (7) can be changed by associated elements of the matrix like (1). Then instead the exact equation (7) we obtain the approximate expression

$$
\left[\begin{array}{cccc}
r_{1,0} & r_{1,1} & \cdots & r_{1, p-2} \\
r_{2,0} & r_{2,1} & \cdots & r_{2, p-2} \\
\vdots & \vdots & \ddots & \vdots \\
r_{p-1,0} & r_{p-1,1} & \cdots & r_{p-1, p-2}
\end{array}\right] \cdot\left[\begin{array}{c}
\alpha_{0} \\
\alpha_{1} \\
\vdots \\
\alpha_{p-2}
\end{array}\right] \cong\left[\begin{array}{c}
r_{0, p-1}-\alpha_{p-1} r_{1, p-1} \\
r_{1, p-1}-\alpha_{p-1} r_{2, p-1} \\
\vdots \\
r_{p-2, p-1}-\alpha_{p-1} r_{p-1, p-1}
\end{array}\right] .
$$

Let $\left[\rho_{i, k}\right]_{i, k=0 \ldots P-2}$ is the inverse matrix with respect to matrix in left part of (8). Then the solution of the system (8) can be presented as

$$
\alpha_{i}=b_{i}-\alpha_{p-1} g_{i}
$$

where $b_{i}=\sum_{k=0}^{P-2} \rho_{i, k} r_{i, p-1} ; g_{i}=\sum_{k=0}^{P-2} \rho_{i, k} r_{i+1, p-1}$. The nonlinear equation (5) generalized for arbitrary correlation matrix has the next manner

$$
\sum_{i, k=0}^{P-1} \alpha_{i} \alpha_{k} r_{i, k} \cong r_{P-1, P-1},
$$

The substitution (9) into (10) gives square equation

$$
a \alpha_{p-1}^{2}+b \alpha_{p-1}+c=0 .
$$

The minimal sensitivity to variations has the extreme of the (11). Therefore as the sought we take the value

where

$$
\alpha_{p-1}=-b / 2 a
$$




$$
\begin{aligned}
& a=\sum_{i=0}^{P-2}\left(\sum_{k=0}^{P-2} g_{i} g_{k} r_{i, k}-2 g_{i} r_{i, p-1}\right)+r_{p-1, p-1}, \\
& b=\sum_{i=0}^{P-2}\left(2 b_{i} r_{i, p-1}-\sum_{i=0}^{P-2}\left(b_{i} g_{k}+b_{k} g_{i}\right)\right) .
\end{aligned}
$$

The numerical experiments have shown that the solution in the form of (9) and (12) corresponds to unitary model of the signal. The element $\alpha_{0}=-a_{p} \cong 1$ with the accuracy $\sim 10^{-10}$.

An arbitrary arrow and column of the target object image matrix can be used for definition of the LSO (2.8) parameters and roots of the characteristic polynomial (2.9) if the image signal is homogeneous. In other casethe components of a $2 \mathrm{D}$ correlation matrix are more informative. The $2 \mathrm{D}$ correlation matrix of the object image region is the following,

$$
\begin{aligned}
& R^{(2)}=\left[r_{i_{x}}^{(2)}, k_{x}, i_{y}, k_{y}\right]_{i_{x}, k_{x}=0 \ldots P-1}^{i_{y}, k_{y}=0 \ldots Q-1}= \\
& {\left[\sum_{m=0}^{n_{x}-P} \sum_{n=0}^{n_{y}-Q_{d^{\prime}}} d_{m+i_{x}, n+i_{y}} d_{m+k_{x}, n+k_{y}}\right]_{i_{x}, k_{x}=0 \ldots P-1}^{i_{y}, k_{y}=0 \ldots Q-1} .}
\end{aligned}
$$

The correlation matrices that characterize data along coordinates in (4.13) may be defined as

$$
\begin{aligned}
& R_{x}^{(2)}=\left[r_{i_{x}}^{(2)}, k_{x}, 0,0\right]_{i_{x}, k_{x}=0 \ldots P-1}^{i_{y}, k_{y}=0} ; \\
& R_{y}^{(2)}=\left[r_{0,0, i_{y}, k_{y}}^{(2)}\right]_{i_{x}, k_{x}=0}^{i_{y}, k_{y}=0 \ldots Q-1} .
\end{aligned}
$$

These matrices can be used as $R_{p}$ in expression (1) for the estimation of two LSO parameters.

\section{The 2D Correlation Matrix Splitting Method (CMSM)}

When texture is dynamic the correlation matrices (1), (14) do not reflect the dynamic nature of the image, so the 2D correlation matrix (13) should be used for estimation of the texture resonant frequencies. The methods of 2D EHD mentioned above differ by data type employed. The MUSIC and ESPRIT methods use second data moments. The MPM method uses first data moments. 2D LPM uses first and second data moments. The 2D EHD method is based on first moment data shift of the dynamic model, which transforms the data along coordinates. We will consider a generalization of these approaches on the platform of the SVD. This decomposition allows select a principal part of an image signal and omit weak fluctuations. Also, it allows split data on mutually shifted matrix pencils in the orthogonal vector space. The matrix pencils create an eigenvalues problem that can be solved by the TLS approach. The eigenvalues are equal to roots of polynomial (2.9). The eigenvalues problem may be written for 2D data $d_{m, n}$ of size $M \times N$ as matrix pencils $[45,46]$ :

$$
D_{x}^{(2)}-z_{x} D^{(2)} ; D_{y}^{(2)}-z_{y} D^{(2)},
$$

where 


$$
\begin{aligned}
& D_{x(y)}^{(2)}=\left[D_{i+k+x}^{(y)} \quad \begin{array}{l}
k=0 \ldots L-1 \\
i=0 \ldots M-L-1
\end{array},\right. \\
& D_{i+k+x}^{(y)}=\left[d_{i+k+x, m+n+y}\right]_{n=0 . . . N-L-1}^{m=0 . L-1},
\end{aligned}
$$

$z$ is the spectral parameter, and $L$ is the splitting parameter. If the $2 \mathrm{D}$ data include $P / 2$ real harmonics and do not contain noise, value $P \leq L$, the rank of matrix pencils (15) is equal to $P$, the data can be presented by $P$ vectors of the SVD. If $z_{x}$ and $z_{y}$ are equal to the matrix pencils eigenvalues, then the expressions (15) are equal to zero. In order to solve the eigenvalues problem the following matrices can be used relatively to $x$ and $y$ coordinates.

$$
Z_{E x(y)}^{(2)}=\left(D^{(2)}\right)^{\#} D_{x(y)}^{(2)}
$$

The SVD can be used for the operation of pseudoinverse in (16) by using of the Plargest singular values and corresponding them eigen vectors. As we mentioned above, the textured image object dynamic model needs in unitary shift transform by the matrix in left part (16) as well as by LSO (2.8). This condition may be supported by including into (16) direct and backward shift transforms to eliminate influence of the dumping factors of the spectral parameters in the case of data matrices splitting. The data matrices in (16) can be accomplished by inverse direction components as $\left[D^{(2)} J D^{(2)}\right]$, where $J$ is the matrix with unit cross-diagonal.

Let multiply the matrices in the left side of (16) by transposed matrices and by such way find the correlation matrix $R^{(2)}=\left[D^{(2)} J D^{(2)}\right]^{T}\left[D^{(2)} J D^{(2)}\right]$. Its SVD can be written as the follows.

$$
R^{(2)}=U \cdot \Xi \cdot V^{T}
$$

where $U$ and $V$ are the matrices of the left and right orthogonal eigen vectors, $\Xi$ is the diagonal matrix of the singular values. Using expression (31) matrix (30) may be rewritten as

$$
Z_{E x(y)}^{(2)}=\left(U_{0}^{H} U_{0}\right)^{-1} U_{0}^{H} U_{x(y)}
$$

where matrices $U_{0}, U_{x(y)}$ have $M$ vectors which correspond to $M$ largest singular values in decomposition (17). Their rows correspond to reciprocally shifted matrices $D^{(2)}$ and $D_{x(y)}^{(2)}$, The matrix $U_{0}$ is the basis matrix of expression (18), the shifted matrix $U_{x(y)}$ has structure that depends on thedirection of the shift: toward $x$ yields $U_{x}$; toward $y$ yields $U_{y}$. The basic matrix $U_{0}$ is 
formed by extracting from matrix $U$ the last $M-L-1$ rows and each $N-L-1$ rows starting from the last row. The matrix $U_{x}$ is formed by extracting from matrix $U$ in (17) the first $M-L-1$ rows. The matrix $U_{y}$ is formed by each $N-L-1$ row of matrix $U$, starting from the first row. The inversion of the matrix $U_{0}^{H} U_{0}$ may be executed by iteration operation [30],

$$
F_{k}=F_{k-1}+\frac{\left(F_{k-1} \mathbf{u}_{\alpha}^{H}\right)\left(\mathbf{u}_{\alpha} F_{k-1}\right)}{1+\mathbf{u}_{\alpha} F_{k-1} \mathbf{u}_{\alpha}^{H}}
$$

where $E_{0}=I_{M}$ is the identity matrix of size $M$, and $\mathbf{u}_{\alpha}$ isthe vector of $\alpha$ th row of the matrix $U$ first $M$ vectors, the indices $\alpha$ denote the set of rows that extracted as was described above. If all extracted rows are used sequentially in (19), then the last matrix $F_{k}$ is equal to $\left(U_{0}^{H} U_{0}\right)^{-1}$. The eigenvalues $z_{x i}, z_{y i}(i=1 \ldots M)$ can be defined as eigenvalues of the matrices (15), (16), (18) which are modified depending on shift direction.

As it follows from the method description, the number of principal harmonic components of the textured image signal EHD is defined by the rank of the matrices in (16). The SVD allows to define harmonics number carefully if $L \geq M \geq P$. The chosen $M$ vectors correspond to largest singular values and therefore the method is not sensitive to phase brakes and noise spikes which usually relate with vectors of the small singular values. This property of the method confirms the earlier made conclusion that alignment with texture EHD is equivalent to the SVD and the EVD.

\section{B. Creation of a Function Basis for High Order EHD}

The matrix operator of the DFT of size $N \times N$ can be presented as a multiplication of simpler matrices on condition that $N$ is a composite number, for example $N=m^{n}$, where $m, n$ are arbitrary integer constants. The factorized presentation gives the algorithm of Fast Fourier Transform (FFT) [30,39]. As it is presented in [48]

$$
\begin{gathered}
F_{N}(k, x)=\prod_{i=1}^{n} W_{i}(k, x)=\prod_{i=1}^{n}\left(V_{m}\left(v_{i}\right) \otimes I_{m^{n-i}}\left(k^{i}, x^{i}\right)\right) \otimes\left(\Omega_{m}\left(m^{i-1} k^{i}, v_{i}, u_{i}\right) \otimes I_{m^{i-1}}\right) ; \\
k=k^{i}+\sum_{j=1}^{i} v_{j} m^{n-j} ; \quad x=m^{i} x^{i}+\sum_{j=1}^{i} u_{j} m^{j-1},
\end{gathered}
$$

where $I_{m^{n-i}}\left(k^{i}, x^{i}\right)$ is a unitary diagonal matrix with numbered rows and columns corresponding to $k^{i}$ and $x^{i}, k^{i}, x^{i}=0,1, \ldots, N / m^{i}-1$. The matrix $I_{m^{n-i}}$ index means its size, $u_{i}=0,1, \ldots, m-1, x^{i}$ are the numbers of data vector elements which are multiplied by the phase rotating matrix

$$
\Omega_{m}\left(k^{i}, v_{i}, u_{i}\right)=\left[\exp \left(-\imath \frac{2 \pi}{N}\left(k^{i}+v_{i} \frac{N}{m}\right) u_{i}\right)\right],
$$


where the indices $v_{i}=0,1, \ldots, m-1$ together with $k^{i}$ are the number of result vector elements. The factorization (20) can be generalized for any $N$ that is a product of arbitrary integer values. If $N=p \cdot m^{n-1}$ the matrix (21) for $i=1$ in (20) is follows:

$$
\Omega_{p}\left(k^{1}, v_{1}, u_{1}\right)=\left[\exp \left(-\imath 2 \pi\left(\frac{v_{1} u_{1}}{p}+\frac{k^{1} v_{1}}{N}\right)\right)\right] .
$$

The first term of the sum in the exponent factor in (22) creates elements of the DFT operator of size $N=p$. The second term is the modulation coefficient which serves to extend low order DFT.

The DFT operator in the base of texture eigen harmonics is presented by matrices $Z_{x(y)}^{\#}$, $Z_{x(y)}$ in (3.6), (3.7). We can extend these matrices by such way as is extended phase rotating matrix (21) in the Fourier operator (20). For this let change the first term of the sum in (22) by elements which are analogues to the matrix $Z_{x(y)}$. Then we will obtain the following phase rotating matrix:

$$
\Omega_{z p}\left(k^{1}, v_{1}, u_{1}\right)=\left[z_{v_{1}+1}^{u_{1}} \exp \left(-\imath 2 \pi \frac{k^{1} v_{1}}{N}\right)\right]_{u_{1}, v_{1} 0, \ldots, p-1}
$$

The substitution of the matrix (23) into the DFT equation (20) yields the operator of DFT with the eigen kernel $(\mathrm{EK})$ associated with the textured object image signal. This signal is characterized by series of resonant frequencies $f_{1} \ldots f_{p}$. Let's write this equation in factored form.

$$
\begin{aligned}
& F_{z N}(k, x)=\prod_{i=2}^{n} W_{i}(k, x) \cdot W_{z}(k, x) ; \\
& W_{z}(k, x)=\left(I_{m^{n-1}}\left(k^{1}, x^{1}\right) \otimes \Omega_{z p}\left(k^{1}, v_{1}, u_{1}\right)\right) ; \\
& \left\{\begin{array}{l}
i=1: k=k^{1}+v_{1} m^{n-1} ; x=p x^{1}+u_{1} ; \\
i>1: k=k^{i}+\sum_{j=1}^{i} v_{j} p m^{n-j-1} ; x=p m^{i-1} x^{i}+\sum_{j=1}^{i} u_{j} p m^{j-2} .
\end{array}\right.
\end{aligned}
$$

The DFT (24) is equivalent to $F_{z N}=F_{N / p} \otimes Z_{p}$. The inverse DFTEK is similar to (24) with phase rotating matrix:

$$
\Omega_{p}^{-1}\left(k^{1}, v_{1}, u_{1}\right)=\left[\bar{z}_{v_{1} u_{1}} \exp \left(l \frac{2 \pi k^{1} v_{1}}{N}\right)\right]_{v_{1}, u_{1}=0, \ldots, p-1},
$$

where $\bar{z}_{v_{1} u_{1}}$ are the elements of a matrix $Z^{\#}$ like the matrices in (3.7) of size $p \times p$. 
The DFTEK implements the PCA and EMD approaches. The $p$ main components can be selected using the EHD and they can be considering as principal components. These $p$ empirical modes are splintered on $N / p$ aliquot quasi-harmonic components and together they create the DFTEK. The textured image signals usually are quasi-periodical, but not exactly harmonic. Therefore the DFTEK spectrum is numerically valid and informative. So, the application of the DFTEK for inverse problem solution and high-order filtering is well-founded. The DFTEK is not exactly orthogonal and has a partly multiplicative functions basis.

\section{Implementation and Experimental Analysis of the IRF}

The IRF can be realized in spatial domain using the convolution of image data with transient characteristic (3.12) or with obtained by the transform (3.8) optimized characteristic (3.20). This operation needs $P \times Q$ multiplications and accumulations per pixel. Another approach is based on filtration in the spectral domain. This approach may be significantly more effective but it has some particularities which will be considered bellow.

Filtration in spectral domain includes definition an image fragment of the size $P \times Q$ with spectrum $A$ in accordance to equation (3.7). The fragment is then multiplied using the elements of the filter spectrum (3.11) or (3.20), and then the filtered values are calculated according to the approximation equation (3.6). These operations need $2(P+Q)+1$ complex MACC operations per pixel. Therefore the filtration in spectral domain has higher efficiency in comparison with the filtration in spatial domain when filter order $P(Q) \geq 64$.

We will consider IRF realization in spectral domain using the example of textured image with foreign object of the size $288 \times 768$ pixels in Fig. 1 . The aim is to recognize the texture of dried up riverbed and remove it. The accompanying problem is to detect the foreign object. One color component of the image signal is presented in Fig.2. As it seen in the figure, the signal amplitude of the foreign object is analogous to the background signal and differs by another dynamic and statistic features. The analysis of inverse correlation matrices [47] has shown that IRF order can be chosen as $P \times Q=32 \times 32$. The base region of size $n_{x} \times n_{y}=64 \times 64$ was chosen in the upper left corner of the image for the IRF transient characteristic evaluation. The result of filtration is the surface presented in Fig. 3. The figure shows that the surface has variation in the neighborhood of $E=1$ as it is presented in (3.3). Because of background texture heterogeneousthere is a surface displacement which becomes lager in the corner opposite to the base region. Signal in the region of the foreign object is clearly observable due to its essential difference from the background. Statistical analysis of the filtered signal was made using the dispersion of the filter (3.3) error noise in the object base region. It was estimated as

$$
\sigma_{\varsigma}^{2}=\frac{1}{n_{x} n_{y}} \sum_{i=0}^{n_{x}-1} \sum_{j=0}^{n_{y}-1}\left(\tilde{d}_{i, j}-E\right)^{2}
$$

where $\tilde{d}_{i, j}=E+\varsigma_{i, k}$ represents the filtered image signal values. The dispersion (1) is the parameter of the target textured object pattern as well as the transient characteristics of the IRF (3.12) or (3.20). 
We can generate an image $G: d_{i, k}^{(t)} \in G ; t=1,2,3$ by the filtration of the image $D: d_{i, k}^{(t)} \in D$ by the IRF (3.3) which contains foreign objects using the following logical filter:

$$
\begin{aligned}
& \text { if } \bigcup_{t=1}^{3}\left(\left|\tilde{d}_{i, k}^{(t)}-E\right|>3 \sigma_{\varsigma}^{(t)}\right) \\
& \text { then } g_{i, k}^{(t)}=d_{i, k}^{(t)} \\
& \text { else } g_{i, k}^{(t)}=0 ; t=1,2,3 .
\end{aligned}
$$

The top indices in the brackets point to color components. The dispersion (1) was estimated for each color component. The filtered image is presented in Fig. 4. As a result of the deviation of image pattern, the image $G$ includes the foreign object, and error fragments of the background that differ slightly from the base region pattern. The appearance of error fragments is also caused by the image fragment spectrum (18) differing in phase from the base region spectrum. The phase equalization of fragments spectrum can be performed in order to eliminate the error objects.

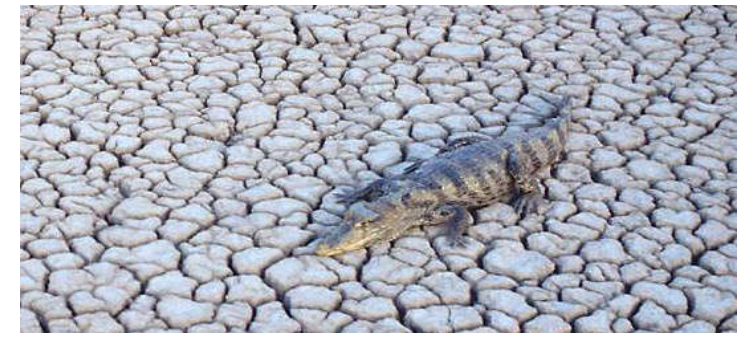

Fig. 1. Textured image background with foreign object

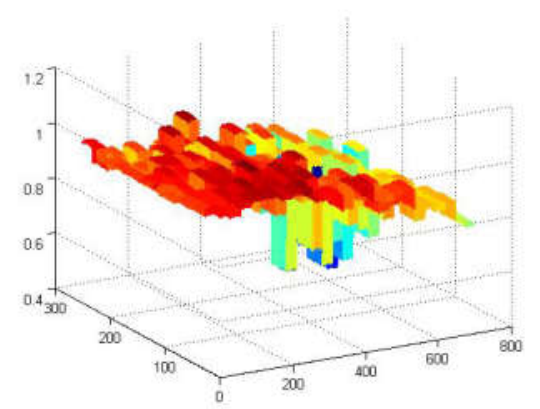

Fig. 3. Image signal filtered by the IRF.

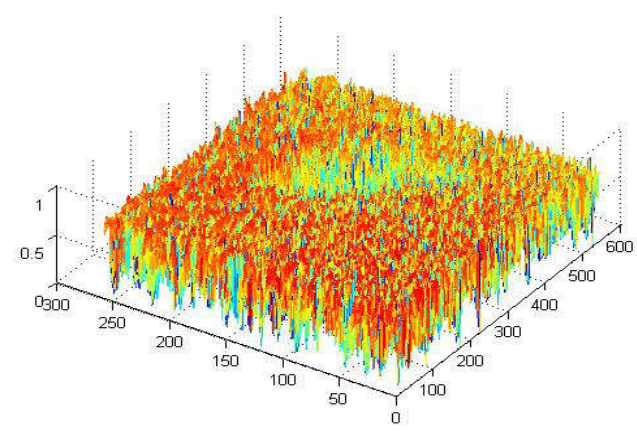

Fig. 2. Image signal of textured background

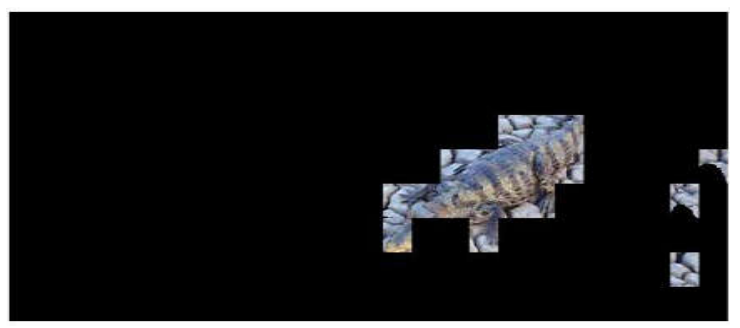

Fig. 4. Filtered texture image with recognized foreign object

Let the spectrum matrix (3.7) of the object base region is the $A^{(0)}$. The approximation (3.6) of some shifted fragment can be written using phase displacement functions as it is shown in (2.11). 


$$
D^{\prime}=Z_{x} \operatorname{diag}\left[e^{\phi_{x 1}} \ldots e^{\phi_{x P}}\right] A^{(0)} \operatorname{diag}\left[e^{\phi_{y 1}} \ldots e^{\phi_{y Q}}\right] Z_{y}^{T}
$$

The phase coefficients in (3) can be found using the least square method from the condition of minimization of the following functional:

$$
\mathrm{I}=\underset{\Phi}{\operatorname{argmin}} \sum_{i=1}^{P} \sum_{k=1}^{Q}\left(A_{i, k} \Phi_{x i} \Phi_{y k}-A_{i, k}^{0}\right)^{2}
$$

where $\Phi_{i}=\exp \left(\phi_{i}\right)$. The variation of the functional (4)

$$
\frac{\partial \mathrm{I}}{\partial \Phi_{x(y) i}}=0 ; i=1 \ldots P(Q)
$$

gives the system of nonlinear equations

$$
\begin{gathered}
\Phi_{x i}=\frac{\sum_{k=1}^{Q} A_{i, k} A_{i, k}^{(0)} \Phi_{y k}}{\sum_{k=1}^{Q} A_{i, k}^{2} \Phi_{y k}^{2}} ; i=1 \ldots P \\
\Phi_{y k}=\frac{\sum_{i=1}^{P} A_{i, k} A_{i, k}^{(0)} \Phi_{x i}}{\sum_{i=1}^{P} A_{i, k}^{2} \Phi_{x i}^{2}} ; k=1 \ldots Q .
\end{gathered}
$$

This system can be simply solved using the iterative method. The initial values of the phase displacement functions in the right part of both equations (5) are trivial: $\Phi_{x(y) i}^{(0)}=1$. The first and next iterations give the sequence of values $\Phi_{x(y) i}^{(k)}, k=1,2, \ldots$. If this sequence agrees with the condition

$$
\sum_{i}\left|\Phi_{x(y) i}^{(k)}-\Phi_{x(y) i}^{(k-1)}\right| \approx \sum_{i}\left|\Phi_{x(y) i}^{(k-1)}-\Phi_{x(y) i}^{(k-2)}\right|
$$

the iteration process can be stopped. The resulting values of $\Phi_{x(y) i}^{(k)}$ are using for spectrum redefinition as it was done in (3). If condition (6) is not met the system (5) has no solution and the phase multipliers are not using.

The image signal filtered by the inverse filter with phase equalization accordingly to (3) - (6) is presented in Fig. 5. In comparison with the signal in Fig. 3, the signal surface is flatter but it has chaotic spikes. That is why the target object image in Fig. 6 is recognized without substantial error zones. Though, it has solitary chaotic error pixels. From the view of object classification such errors are less dangerous than those that are in Fig.4. They can be ignored. No more than 5 iterations were used to solve equation system (5). 


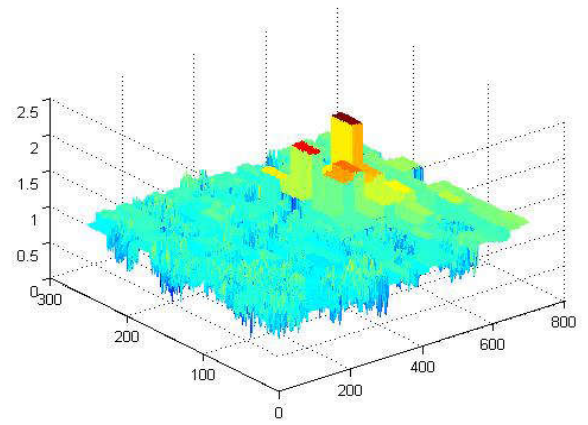

Fig. 5. Image signal filtered by the inverse filter with spectrum phase equalization

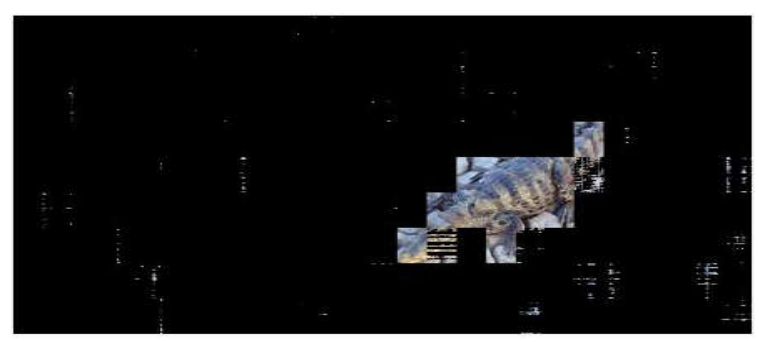

Fig. 6. Object image filtered by the inverse filter with spectrum phase equalization.

Fig. 4 and Fig. 6 show that filtration in spectral domain has some deficiencies. Image zones can be recognized with some space step. This reduces resolution ability of the recognition. Therefore the filtration in the spectral domain may be considering as a fast and rough estimator of the target object boundaries. The object boundaries can be refined by post-filtering of the recognized and adjoined zones using the same IRF in the spatial domain. The results of postfiltering of nontrivial zones of the images in Fig. 4 and in Fig. 6 are shown in Fig. 7 and Fig.8.

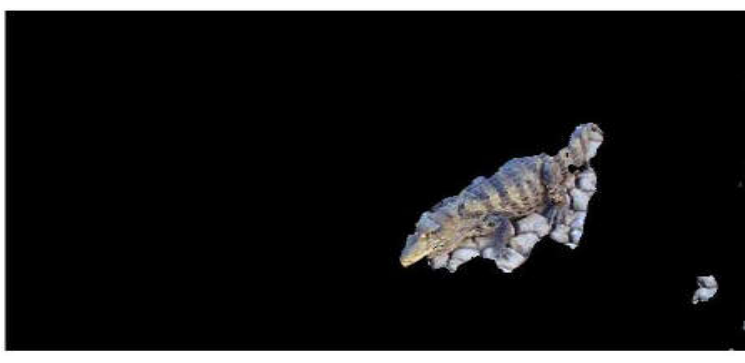

Fig. 7. The result of image post-filtration in spatial domain of the image in Fig.4
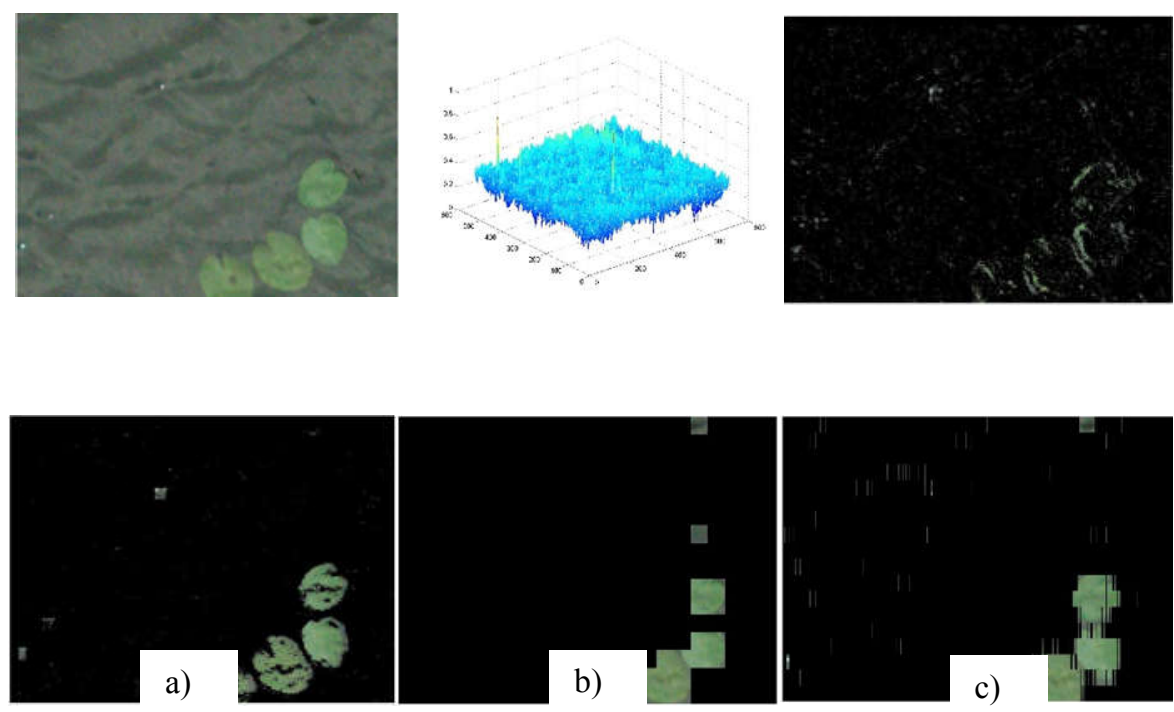

e)
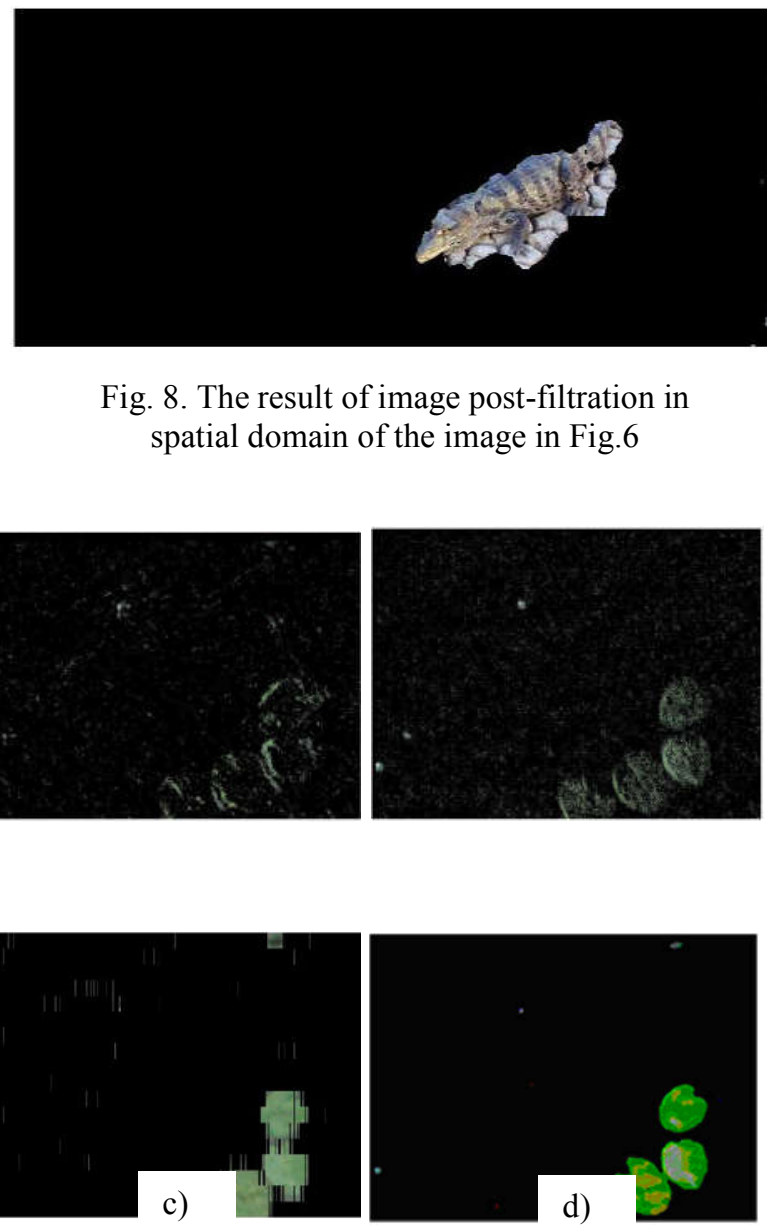

Fig. 8. The result of image post-filtration in spatial domain of the image in Fig.6

g)

h)

Fig. 9. Initial image a), one color signal b), the results of filtration by AR c), by AR with shifted mean (3.27) d), by IRF (3.12) e), by IRF in spectral domain f), by IRF in spectral domain with phase equalization $\mathrm{g})$, by the post filtration by the optimal IRF (3.20). 
The example in fig. 9 demonstrates ability of the IRF to recognize dynamic texture and sensitivity to small objects. The texturein Fig. 9a) can be considering as spatial dynamic type because each its fragment isa result of a dynamic change or extension of an adjacent fragment. Fragments are similar but not identical. This is evident in Fig 9b). The foreign objects signal slowly differs from the background texture signal. The Fig. 9c)-d) shows that alternative to the IRF usual AR filter and AR filter with shifted mean (3.27) of $16^{\text {th }}$ order give dissatisfied results. As it seen in Fig. 9e), the IRF (3.12) of $16^{\text {th }}$ order allows to recognize target texture as well as small (white points) and slow foreign objects. It was used the region of size $64 \times 64$ in the left high corner of the initial image for evaluation of the filters parameters. Fast filtration in the spectral domain (Fig. 9f)g) gives same results but with rough objects boundaries and some errors. The result of the post filtration of selected and adjoining zones of the image by optimal IRF (3.20) is shown in Fig. 9h). The filter allows to specify foreign objects boundaries and eliminate mistake objects in all color components.

The example in Fig. 10 shows implementation of high order filtration with using of DFTEK. The multi-component image in Fig. 10a) of size $2496 \times 3072$ pixels includes the target textured background composed of wheat grain. There are zones of wet grain, grains of oats and rye with fuzzy boundaries within the image. A filter order of $P \times Q=96 \times 96$ was used for texture recognizing. The filter order was decomposed as $P(Q)=p \cdot m^{n}=6 \cdot 2^{4}$. For generation of the DFTEK basis (4.24) where used three pairs of complex roots of the characteristic polynomial (2.9) of $96^{\text {th }}$ order which correspond to maximal amplitudes of the spectrum (3.7). Results of the filtration are presented in Fig. 10 b)-d). They have the same properties as the previous examples. The results of the filtration with using the EHD of $96^{\text {th }}$ order have same quality but it needs up to 7 times more MACC complex operations.

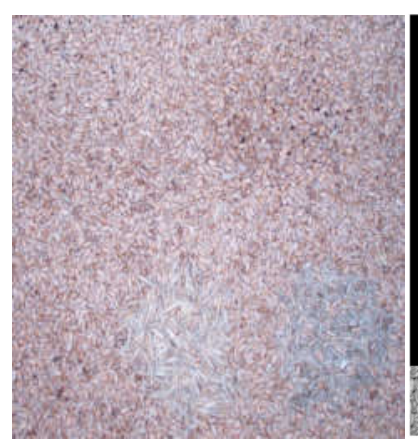

a)

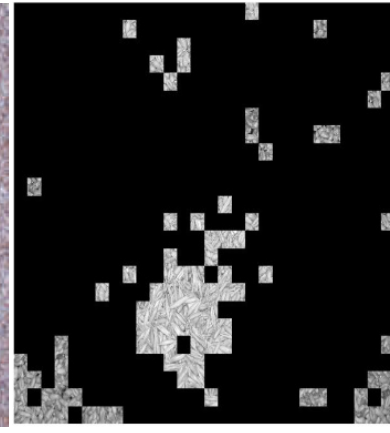

b)

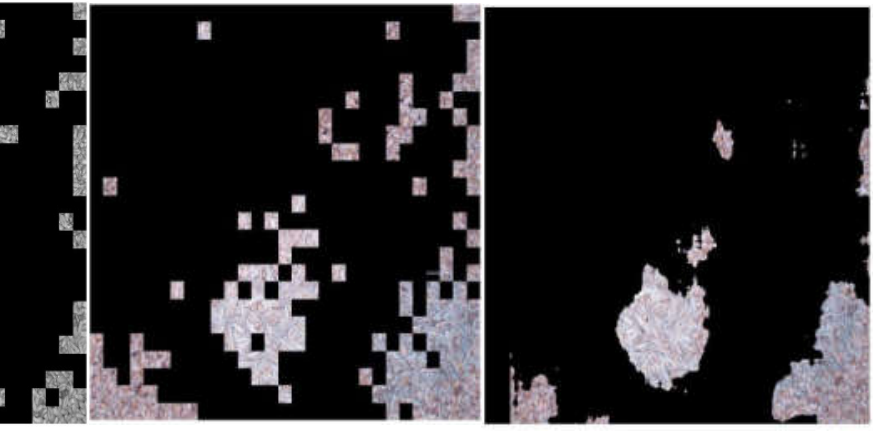

c) d)

Fig. 10. Object recognition using DFTEK. Initial multi-component image a), image filtered in spectral domain b), in spectral domain with phase alignment c), post-filtration of previous image in spatial domain d).

The LS (4.3) of correlation matrices (4.14) was used for LSO (2.8) parameters estimation as well as EHD basis synthesis in the caseof the first two examples. The method 2D CMSM was used to estimate the 96 principal harmonic components of the target texture model.

The bank of IRF can be used for classification of textured components of the multicomponent image. The example of the filter bank is shown in the Fig. 11. Three filters of $16^{\text {th }}$ order are designed for corresponding textured images in Fig. 11b), d), f) and was used for filtration of the initial image in Fig. 11a). The results of the optimal filtration are shown in Fig. 11c), e), g). 
As it seen from Fig. 11, the target textured objects are recognized and image in Fig. 11a) can be decomposed on separated components.

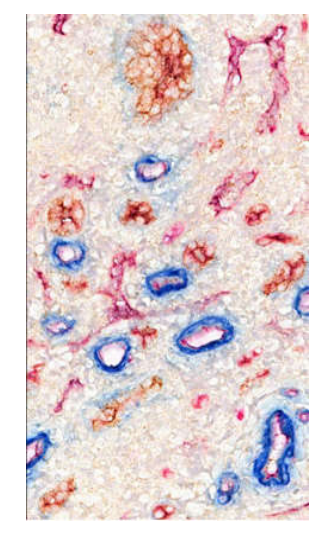

a)

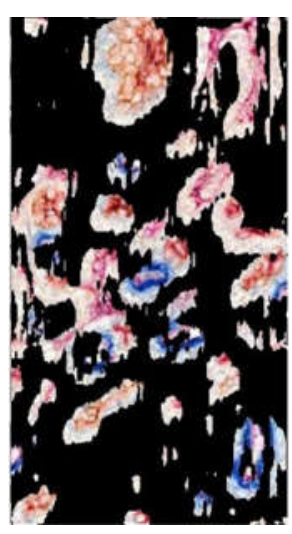

c)

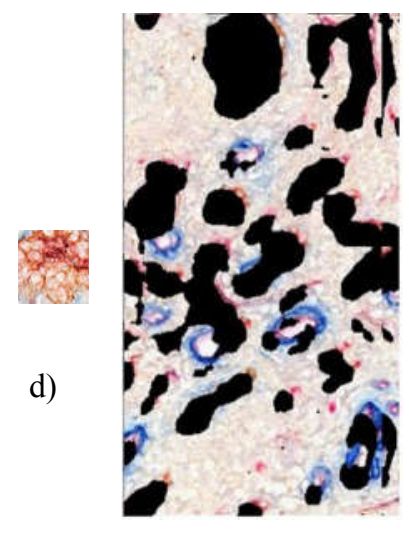

e)

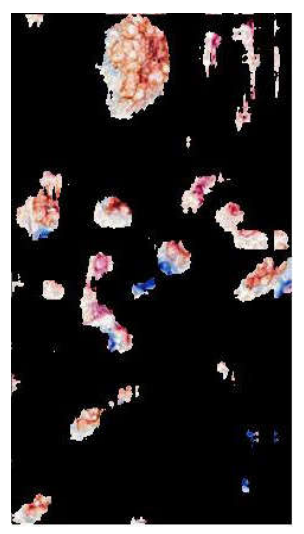

g)

Fig. 11. Filtration of the multicomponent texture a) by the IRF bank of the textures b), d), f) which gives the result images c), e), g) where the corresponding textures are eliminated.

When texture is varying in time and space and is characterizing by non-stationary surges the application of simple statistical analysis and logical filtration as in (1) - (2) will not provide high quality filtration and recognition. If texture tracking is continuous it is possible to remove false foreign objects by binary correlation of the objects that are found in consequent frames.

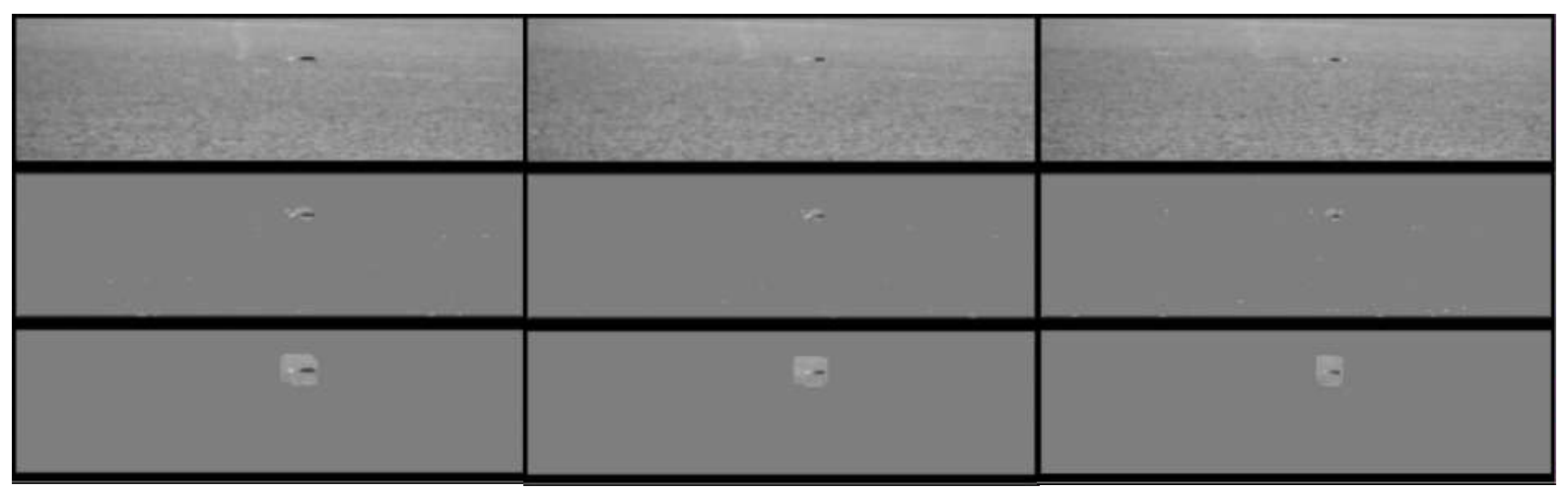

Fig. 12. Three consecutive fragment of the dynamic time and space varying texture as the marine surface (high row) are filtered by the IRF (middle row) and correlation filter (5.7) (low row) for robust recognition of the foreign object.

The method of filtration by binary correlation includes [49]:

1) Estimation of the objects size and its geometrical center.

2) Evaluation of the correlation coefficients between objects in $L$ consecutive frames:

$$
r=\frac{\sum_{t=0}^{L-1} \sum_{i, j \in S_{x} \times S_{y}}\left\{g_{i, j}^{[0]}>0\right\}^{\wedge}\left\{g_{i, j}^{[t]}>0\right\}}{\sum_{t=0}^{L-1} \sum_{i, j \in S_{x} \times S_{y}}\left\{g_{i, j}^{[t]}>0\right\}}
$$


where $\left\{g_{i, j}^{[0]}>0\right\}^{\wedge}\left\{g_{i, j}^{[t]}>0\right\}$ denotes the logical function “ $A N D$ ”. If the condition in the brackets is true then the value is equal to one, otherwise the value is zero. $S x \times S y$ is the object location. The numbers in the square brackets point to the frame number.

3) If the correlation coefficient (7) exceeds the threshold level then the object is true, otherwise, it is false.

4) A true object is refined with its immediate surrounding background for further classification.

5) The $L$ frames running time should be much smaller in comparison to the moving time of the object of interest within the location rectangle $S x \times S y$.

Consecutive frames of marine surface filtering are shown in Fig. 10. As it seen in high row, the texture of marine surface is heterogeneous and therefore the filtration was made by filters bank of fore IRF for fore separated image zones along vertical coordinate. Full frame size is $200 \times 600$ pixels. A base region of size $32 \times 32$ for design the IRF of $8^{\text {th }}$ order in each zone was chosen in its upper left corner.It was used theCMSM. The binary correlative filter (7) with parameters $L=3$ and $r=0.3$ was used. As it seen in middle row of Fig. 10, some errors remained after IRF and logical filtration (2), these zones were removed successfully with the help of correlative filtration (7).

\section{Conclusion.}

Analysis of methods of textured image modeling has shown that current researches focus on the decompositions and integral transforms that provide the most informative parameters and reflect the spatial and temporal properties of the texture. These methods are founded on the symmetry of a texture in respect to shift and rotate transforms. The symmetry means that exist character of a texture pattern transform which reflects its changes in space or time. This type of dynamic transform can be represented by the linear shift operator that models step-type changes of texture pattern as well as its periodicity. The eigenvectors of the LSO can serve as a basis for eigenharmonic decomposition of the texture with invariance to shift transforms.

The problem of object recognition against a textured background can be solved by elimination of the background by the IRF if the background is presented as a response of the dynamic model on exciting signal of simple form. The classification of the exiting signal pattern is sufficiently simpler than classification of the original image.

We have shown in (1.14) that if the inverse filtration is resonant in respect to texture pattern than such filter is optimal. The term "resonant" has many means. In general, this is equivalence of a target object to some templates of pattern. The resonance with harmonic fluctuations is mostly known.

The next step of the filtration optimization is evaluation of the optimal transient characteristic (3.20) in spectral domain. Although the EHD basis provides a mathematically correct solution of the inverse problem with a certain number of estimated harmonic principal components, the elements of the amplitude matrix in (3.7) and their inverse values are sensitive to empirical data chaotic fluctuations. Therefore the optimization of the found spectrum (3.12) needs for enforcing of main harmonic components. This is achieved by the help of additional restrictions on the form of the spectral matrix in (3.16) - (3.18). The restriction of minimal surface allows select principal spectral components. It was need up to 5 iterations (3.20) to achieve the condition (3.23). The next step of the optimization (3.24) is rather a verification of the transient characteristic inversion. The condition (3.26) is satisfied after 2 iterations (3.25).

The couple of transforms (3.6) and (3.7) play the key role in the filter design. They relay with careful estimation of the LSO and its eigenvalues which mean the parameters of the texture pattern 
eigen fluctuations. The numerical experiments have shown that the IRF carefully eliminates fluctuations if the series (3.6) approximates the texture surface with error level not exceed $-40 \mathrm{~dB}$ in respect to image signal power. This criterion can serves for choosing of the method of harmonic decomposition, correlation matrices (4.13) or (4.14), target of approximation - original image or its reflexes in the manner of flat plane projection, curvature or Laplace-Beltrami flow and others.

The inverse filter designed and implemented using EHD of the texture acquires the same invariance to space transforms that the EHD provides. Therefore the filtration in spectral domain is preferable for high order filtration.Foreign object presence in a texture fragment corrupts an arbitrary number of the pattern spectral components. So, the filtration in spectral domain resolution ability is determined by fragment size which is equivalent to filter order. But, as it was shown by computation examples, the resolution can be improved by optimization of resonant interaction of the filter and texture fragments by phase aligning (5.3). The phase alignment is unitary operation which does not change image signal power. This procedure also reduces sensitivity of the filter to weak variations in the textured image structure.

The filtration in spectral domain was optimized in respect to number of computations by generation of the DFTEK basis in the manner of factorized fast transform (4.24). The using of the DFTEK instead of the usual EHD does not essentially change the quality of filtration. The computational advantage depends from the DFTEK structure and can be significant in comparison with the EHD.

Also, we offer the optimized schema of the objects recognition. The schema includes the next stages:

- fast rough filtration in spectral domain;

- statistical analysis and selection nontrivial zones;

- the filtration in space domain using convolution with transient characteristic of the nontrivial zones and adjacent ones to them;

- statistical analysis of the obtained signal for exact texture bound recognition.

The considered above method of textured image filtration and foreign object recognition differs from other known method by following features: each texture is characterized by unique own filter; it is feasiblefilter implementation in real time using graphic processor units;regulation of resolution and quality is provided; invariance to image movement induced changes;based on principal harmonic decomposition that is more convenient than the EMD, or the EVD, because it has an analytical form, and may be adapted to any image and image sample fragment.

\section{References}

[1] T. Randen and J. H. Husoy, "Filtering for Texture Classification: A Comparative Study", IEEE Trans. Pattern Analysis and Machine Intelligence, vol. 21, no. 4, pp. 291-310, 1998.

[2] R. J. Radke, S. Andra, O. Al-Kofahi, and B. Roysam, "Image change detection algorithms: a systematic survey", IEEE Trans. onImage Processing, vol.14, no. 3, pp. 294-307, 2005.

[3] R. Haralick, K. Shanmugam, and I. Dinstein, "Textural features for image classification", IEEE Transactions on Systems, Man, and Cybernetics, vol. 3(6), pp. 610-621, 1973.

[4] Xianghua Xie, "A Review of Recent Advances in Surface Defect Detection using Texture analysis Techniques", Electronic Letters on Computer Vision and Image Analysis, vol. 7(3), pp. 1-22, 2008.

[5] G. Celeux, F. Forbes, and Peyrard N, "EM procedures using meanfield-like approximations for Markov model-based image segmentation" Pattern Recognition, vol. 36(1), pp.131-144, 2003. 
[6] S. Lazebnik, C. Schmid, and J. Ponce, "Affine-invariant local descriptors and neighborhood statistics for texture recognition", In Proc. ICCV, vol. 6, pp. 221-229, 2003.

[7] Xiangjun Zhang and Xiaolin Wu, "Image Interpolation by Adaptive 2-D Autoregressive Modeling and Soft-Decision Estimation", IEEE Trans. onImage Processing, vol.17, no. 6, pp.887$896,2008$.

[8] M. Sayadi, S. Sakrani, F. Fnaiech, and M. Cheriet, "Gray-level Texture Characterization Based on a New Adaptive Nonlinear Auto-Regressive Filter", Electronic Letters on Computer Vision and Image Analysis, vol. 7(1), pp. 40-53, 2008.

[9] M. Partio, B. Cramariuc, and M. Gabbouj, "An Ordinal Co-occurrence Matrix Framework for Texture Retrieval", EURASIP Journal on Image and Video Processing, Article ID 17358,15 p, 2007.

[10] U. Clarenz, U. Diewald, and M. Rumpf, "Processing textured surfaces via anisotropic geometric diffusion”, IEEE Trans. on Image Processing, vol. 13, no 2, pp. 248-261, 2004.

[11] Yun Fu, T. S. Huang, "Image Classification Using Correlation Tensor Analysis", IEEE Trans. onImage Processing. vol.17, no 2, pp. 226-234, 2008.

[12] Hongliang Li, Guizhong Liu, and Zhongwei Zhang, "A new texture generation method based on pseudo-DCT coefficients", IEEE Trans. onImage Processing, vol.15, no. 5, pp. 13001312, 2006.

[13] K. Huang, S. Aviyente, "Information-theoretic wavelet packet subband selection for texture classification", Signal Processing, vol. 86, pp. 1410-1420, 2006.

[14] C. Palm and T. M.Lehmann "Classification of color textures by Gabor filtering", Machine Graphics\&Vision, vol. 11, no 2/3, pp. 195-219, 2002.

[15] V. S. Vyas, P. Rege "Automated Texture Analysis with Gabor filter", GVIP Journal, vol. 6, no. 1, pp. 35-41, 2006.

[16] N. G. Kingsbury, "Complex wavelets for shift invariant analysis and filtering of signals", Journal of Applied and Computational Harmonic Analysis, vol. 10, no. 3, pp.234-253, 2001.

[17] P. Hill and D. Bull, "Rotationally Invariant Texture Features using the Dual-Tree Complex Wavelet Transform", in Proc. ICIP 2000, Vancouver.

[18] Can Evren Yarman and Birsen Yaz, "An Inversion Method for the Exponential Radon Transform Based on the Harmonic Analysis of the Euclidean Motion Group", Proc. of SPIE 2006, v. 1, 6142, 61424A.

[19] Guangcan Liu, Zhouchen Lin, and Yong Yu, "Radon Representation-Based Feature Descriptor for Texture Classification”, IEEE Trans. onImage Processing, vol.18, pp. 921-928, 2009.

[20] F. Ade, "Characterization of texture by “eigenfilter.", Signal Processing, no. 5, pp. 451$457,1983$.

[21] D. Coltuc, T. Fournel, J. M. Becker, and M. A. Jourlin, "Multiresolution Independent Component Analysis for textile images", Anti-counterfeit Image Analysis Methods: A Special Session of ICSXII IOP Journal of Physics: Conference Series 77, 2007.

[22] A. Khaparde, B. L. Deekshatulu, M. Madhavilatha, Z. Farheen, and S. Kumari, "Content Based Image Retrieval Using Independent Component Analysis", IJCSNS International Journal of Computer Science and Network Security, vol.8, no. 4, pp. 327-332, 2008.

[23] J. C. Nunes, O. Niang, Y. Bouaoune, E. Delechelle, and P. Bunel "Bidimensional Empirical Mode Decomposition Modified for Texture Analysis", in Proc. 13th Scandinavian Conference, SCIA 2003, Halmstad, Sweden, June 29 - July 2, pp. 171-177, 2003.

[24] Xiong Chang-zhen, Xu Jun-yi, Zou Jian-cheng, and Qi Dong-xu, "Texture classification based on EMD and FFT", Journal of Zhejiang University SCIENCE, vol. 7(9), pp. 1516-1521, 2006. 
[25] Z. Zhihua Yang and L. Yang "Decomposition Based on Classical Empirical Mode Decomposition and Radon Transform", in Proc. Of IMECS 2009, March 18 - 20, 2009.

[26] M. E. Wall, A. Rechtsteiner, and L. M. Rocha, "Singular value decomposition and principal component analysis", in A Practical Approach to Microarray Data Analysis, Norwell, MA, 2003.

[27] S. Soatto, G. Doretto, and Y. N. Wu, "Dynamic Textures “, International Journal of Computer Vision, vol. 51, pp. 91-109, 2003.

[28] R. Costantini, L. Sbaiz, and S. Susstrunk, "Higher. Order SVD Analysis for Dynamic Texture Synthesis", IEEE Trans. onImage Processing, vol. 17, no 1, pp.42-52, 2008.

[29] K. Fujita and Sh. K. Nayar, "Recognition of Dynamic Textures using Impulse Responses of State Variables", Pattern Recognition, vol. 31, pp. 1496-1509, 2003.

[30] S. L. Marple, "Digital Spectral Analysis with Applications", Prentice-Hall, Inc., Englewood Cliffs, New Jersey, 492 p., 1987.

[31] Y. Huang and K. L. Chan, "Texture decomposition by harmonics extraction from higher order statistics", IEEE Transactions onImage Processing, vol.13, no. 1, pp.1-14, 2004.

[32] M. S. Pattichis and A. C. Bovik, "Analyzing Image Structure By Multidimensional Frequency Modulation”, IEEE Trans. on Pattern Analysis and Machine Intelligence, no. 5, pp. 136, 2006.

[33] R. Kimmel, N. A. Sochen and R. Malladi, "On the Geometry of Texture". International Conference on Curves and Surfaces [4th], Saint-Malo, France, 1-7 July 1999. Proceedings, Volume 1. Curve and Surface Design.

[34] N. Peinecke, F.-E. Wolter, M. Reuter, "Laplace spectra as fingerprints for image recognition", Computer-Aided Design, vol. 39, 460-476, 2007.

[35] D. Charalampidis, "Texture synthesis: textons revisited", IEEE Trans. onImage Processing, vol.15, no. 3, pp.777-787, 2006.

[36] Yu. A. Bunyak, "Harmonic analysis of wave fields", J. of Communication Technology and Electronics, vol.43, no. 3, pp. 239-243, 1998.

[37] N. Wiener, "Time series", M.I.T. Press, Cambridge, Massachusets, p. 42, 1964.

[38] A. Khintchine, "Korrelationstheorie der stationaren stochastichen prozesse", Matematichen Annalen, vol. 109 (1), p. 604-615, (1934).

[39] D. F. Elliot and R. Rao, "Fast Transforms: Algorithms, Analysis and Applications", Academic Press. New York, 1983.

[40] P. S. Muhly and B. Solel, "Tensor Algebras, Induced Representations, and the Wold Decomposition", Canad. J. Math, vol.51, no. 4, pp. 850-880, 1999.

[41] Gu Xianfeng, Yalin Wang, and Shing-Tung Yau. "Computing Conformal Invariants: Period Matrices", Communications in information and systems, vol.3, no. 3, pp. 153-170, 2004.

[42] M. M. Barbieri, P. A. Barone, "Two-dimensional Prony's method for spectral estimation", IEEE Trans, vol. SP-40, no. 11, pp. 2747-2756, 1992.

[43] Yu. A. Bunyak, "Two dimension spectral analysis on method of maximum likelihood on base of the linear symmetry of correlation matrix", Padioelectronics and Communication systems, vol.39, no. 4, 1996.

[44] S. Rouquette and M. Najim, "Estimation of Frequencies and Damping Factors by TwoDimensional ESPRIT Type Methods", IEEE Trans. on Signal Processing, vol.49, no 1, pp. 128137, 2001.

[45] Y. Hua, "Estimating two-dimensional frequencies by matrix enhancement and matrix pencil”,IEEE Trans, vol. SP-40, no. 9, pp. 2267-2280, 1992.

[46] Yu. A. Bunyak, "Estimation of radiation sources angular coordinates by the method of antenna array data samples matrices factorization", J. of Communication Technology and Electronics, vol.40, no. 1, pp.87-94, 1995. 
[47] O. Bunyak, Yu. Bunyak, "Location and Estimation Parameters of Weak Wave Packets in Noise", IEEE Instrumentation and Measurement Technology Conference - IMTC-2007, Warsaw, Poland, May 1-3, 2007.

[48] Yu.A. Bunyak, "The class of discrete orthogonal sub-exponential functions", J. of Communication Technology and Electronics, vol.34, no. 6, pp.1300-1303, 1989.

[49] Y. Podobna, R. Kvetnyy, J. Schoonmaker, Yu. Bunyak, O. Sofina, C. Boucher, V. Contarino, A cooperative effort to develop a real time marine mammal detection and tracking system, Proc.AUVSI's Unmanned Systems Europe 2009 , La Spezia , Italy, 2009. 